\title{
Discrete time and continuous time formulations for a short sea inventory routing problem
}

\author{
Agostinho Agra · Marielle Christiansen . \\ Alexandrino Delgado
}

the date of receipt and acceptance should be inserted later

\begin{abstract}
We consider a fuel oil distribution problem where an oil company is responsible for the routing and scheduling of ships between ports such that the demand for various fuel oil products is satisfied during the planning horizon. Inventory management considerations are taken into account at the demand side only, and consumption rates are given and assumed to be constant. We provide two alternative mixed integer formulations: a discrete time model adapted from the case where the consumption rates are varying and a classical continuous time formulation. We discuss different extended formulations and valid inequalities that allow us to reduce the linear gap of the two initial formulations. A computational study comparing the various models accordingly to their size, linear gap and running time, was conducted based on real small-size instances, using a commercial software.
\end{abstract}

\section{Introduction}

Maritime transportation is a major mode of transportation of goods worldwide. The importance of this mode of transportation is obvious for the long distance transportation of cargoes but it is also crucial in local economies where the sea is the natural link between the local developed regions, such as countries formed by archipelagoes. When a company has the responsibility of coordinating the transportation of goods with the inventories at the ports, the underlying planning problem is a maritime inventory routing problem. Such problems are very complex. Usually modest improvements in the supply chain planning can translate into significant cost savings.

This is a preprint of the paper published in Optimization and Engineering, 18(1), 269-297, 2017. The final publication is available at link.springer.com

A. Agra

Department of Mathematics and CIDMA, University of Aveiro. E-mail: aagra@ua.pt

M. Christiansen

Department of Industrial Economics and Technology Management, Norwegian University of Science and Technology. E-mail: mc@iot.ntnu.no

A. Delgado

Department of Mathematics, University of Cape Verde. E-mail: alexandrino.delgado@docente.unicv.edu.cv 
In this chapter we consider a real maritime inventory routing problem occurring in the archipelago of Cape Verde. An oil company is responsible for the inventory management of different oil products, such as, diesel, gasoline, fuel and jet, in several tanks located in the main islands. Fuel oil products are imported and delivered to specific islands and stored in large supply storage tanks. From these islands, fuel oil products are distributed among all the inhabited islands using a small heterogeneous fleet of ships. These products are stored in consumption storage tanks. Some ports have both supply tanks for some products and consumption tanks of other products. Not all islands consume all products.

Consumption rates are assumed to be given and constant. Typically the consumption rates are forecasted. Hence, safety stocks must be considered. Additionally, the storage tanks have limited capacity. Therefore, the level of each product in each tank must always be kept between a given lower level, determined by the safety stock, and an upper level, determined by the tank capacity. As the capacity of the supply tanks is very large when compared to the total demand over the horizon, we omit the inventory aspects for these tanks.

To transport fuel oil products between the islands, the planners control a small heterogeneous fleet. Each ship has a specified load capacity, fixed speed and cost structure. The cargo hold of each ship is separated into several cargo tanks. The products cannot be mixed, and cleaning operations to change between products on the same tank should be avoided. Therefore we assume that the ships have dedicated tanks for each product. Each port can receive at most one ship at a time, and in some ports there exist a minimum time interval between the departure of one ship and the arrival of the next ship.

Given the initial stock levels at the consumption tanks, the initial ship position (which can be a point at sea) and the quantities on board each ship, the inter-island distribution plan consists of designing routes and schedules for the fleet of ships including determining the number of visits to each port and the (un)loading quantity of each product at each visit to each port. This plan must satisfy the safety stocks of each product at each island, and the capacities of the ships and tanks. The transportation and operation costs of the distribution plan are to be minimized. This problem is called a Short Sea Inventory Routing Problem (SSIRP). Short sea stands for sea transportation between ports located in the same geographical area, in contrast to deep sea which is typically transportation between continents.

We have witnessed an increased interest in studying optimization problems within maritime transportation. See the reviews on maritime transportation; [12-14]. Combined routing and inventory management within maritime transportation have been present in the literature the last one and a half decades only; see [6] and [10]. These problems are often called Maritime Inventory Routing Problems (MIRPs). Most of the published MIRP contributions are based on real cases from the industry, see for the single product case $[9,16-18,20]$ and for the multiple product case $[5,11,24,25,28-30]$.

In $[5,9,28]$, the production and/or consumption rates are considered given and fixed during the planning horizon. For those problems event based models are used where an index indicating the visit number to a particular port is added to most of the variables. These event based models are known as time continuous models [13]. In [1, 16, 18-20, 24, 25, 29] time discrete models are developed to capture the complicating factors with varying production and consumption rates.

The most related problems to the SSIRP given here are presented in [2] and [5]. In [2] it is considered a variant of this SSIRP for short-term planning with demand orders, that is, amounts of oil products that must be delivered within a given time period. These orders are determined from the initial stock levels and the consumption rates. Typically, demand orders lead to a problem with varying demands where demands are zero for most time periods and 
a large amount for a few periods. Several key issues taken into account in the short-term problem, such as port operating time windows for each time period, are relaxed here or incorporated indirectly in the data. Otherwise, the problems considered originate from the same company in the same region. In [5], a problem similar to the SSIRP is considered with constant consumption rates. However, in [5] only a continuous model is considered. In both papers the products have dedicated compartments in the ships.

In the literature, many of the MIRP studies give a MIP model and use this model in various solution approaches. Arc based models with arc variables representing the sailing between two ports, are used in branch-and-bound and branch-and-cut approaches (e.g. $[1-3,29])$. In [29], subproblems that are restricted versions of the original problem are solved by branch-and-cut. These subproblems are solved iteratively in a large neighbourhood search heuristic. In [28], an arc based model is combined with a heuristic. Finally, the authors in [26] use a rolling horizon heuristic with an underlying arc based model. Path flow models, where the path is described by the route and/or schedule, are used with pre generated paths [19], in branch-and-price methods (e.g. [23] and [16]), rolling horizon heuristics [25] and various fix and relax heuristics [21]. In [19], an arc based model is compared with a path flow model for a liquefied natural gas inventory routing problem. From the limited number of instances run, it was hard to conclude which formulation was superior. The path flow formulation was able to solve more instances faster to optimum than the arc based formulation, while the arc based formulation found the first integer solution faster than the path-flow formulation. Furthermore, the path flow formulation suffered from poor scaling capabilities, so the same problem was solved by a branch-and-price method in [20].

Just recently the study of valid inequalities has been incorporated in MIRPs. In [27] valid inequalities are included in order to enhance the proposed formulations to an oil product transportation problem, and in [23] valid inequalities are developed within a column generation approach for a maritime inventory routing problem. Also, in [16] valid inequalities are derived for a single-product maritime inventory routing, which are used within a branchprice-and-cut algorithm. In [20], valid inequalities are included to improve the formulation presented for the liquefied natural gas inventory routing problem. Finally, [29] presents valid inequalities for MIRPs including several practical constraints for solving problems in different shipping segments. Comparison of different formulations in conjunction with valid inequalities have been used in [1] and [2].

As discussed in both [6] and [29], most combined maritime routing and inventory management problems described in the literature have particular features and characteristics, and tailor-made methods are developed to solve the problems. These methods are often based on heuristics or decomposition techniques. The choice of these solution approaches might be explained by the high complexity of real MIRPs and the possibility to utilize the special structure of the problem. However, the constant hardware development combined with the theoretical advances in optimization techniques have produced optimization solvers capable of handling increasingly larger instances. Currently, it is possible to obtain optimal or near optimal solutions to small real instances occurring in maritime transportation problems using commercial solvers. See [2] for the case of Cape Verde, and [1, 22, 27, 29].

Mathematical formulations, and related discussion, for MIRPs have received some attention during the last decades, see for instance, [1-3, 5, 8, 16, 29]. However, comparison of different formulations for a given MIRP has just been considered in a few studies so far; see e.g. $[1,2,19]$. Such studies are of crucial relevance when planning to solve a problem or subproblems (embedded in a more general solution approach) using commercial solvers. The SSIRP considered here offers an interesting test bed for a computational study of different 
formulations. In this chapter we discuss and compare different mathematical formulations for the SSIRP, some of them sharing the characteristics of well-known and widely used formulations. Therefore, although the problem presented here is a particular maritime inventory routing problem, the formulations discussed and compared are of interest to other related maritime inventory routing problems as well.

In addition to the common approach (see [5] and [9]) that consists of using event based models (known as continuous time models), we introduce a model that combines a discrete and continuous time where the discrete time corresponds to an artificial discretization of the continuous time. This model is similar to the one given in [2] for SSIRP with time varying consumption rates. For each approach, following [2] (see also [1] for a completely discrete model), we develop an arc-load formulation and two extended formulations. Arcload formulations are the most used formulations in MIRPs, see [5, 9, 29]. The extended formulations use new sets of variables that provide additional information about the solution. That information is essential to derive a tighter model, that is, to derive a model whose linear relaxation is closer to the optimal solution than the linear relaxation of the arc-load model. Similar extended formulations have been extensively used for other problems, such as lotsizing and network flow problems. In MIRPs they have been used in [1,2] for problems with time varying consumption rates. To the best of our knowledge, the two extended formulations introduced for the event time model, and the formulations resulting from adaptation to the constant rate problem of models including time discretization, are new for MIRPs.

We provide a comparison of the two approaches and the three different formulations for each approach using as criteria the size of the models, the integrality gaps, the number of branch and bound nodes, and the running time to solve the instances. All formulations are strengthened with valid inequalities and tightening of constraints. As in [1,2], computational experiments indicate that the best performances are obtained using extended formulations based on sets of variables that associate flows to the ship arcs (called arc-load flow models). This conclusion is highly relevant since, as mentioned above, most MIRPs have been modeled using arc-load formulations which are dominated (both theoretically, considering the integrality gap, and computationally, considering the running times) by the arc-load flow models.

The real test instances are of small size which allow us to use a commercial software to solve them to optimality. However, it should be remarked that the tested models have a structure that is well suited for solving instances with longer planning horizons than those considered here. For instance, the underlying models can be used in heuristic procedures such as rolling-horizon heuristics, relax-and-fix heuristics, etc. to derive feasible solutions. In [3], instances are heuristically solved for time horizons of several months using a rolling horizon heuristic where the planning horizon is split into smaller sub-horizons. Then, repeatedly, a limited and tractable problem (which is much related to the one considered in this paper) is solved for the shorter sub-horizons using a commercial software.

The remaining of this chapter is organized as follows. Section 2 presents arc-load discrete time and arc-load continuous time formulations. Extended formulations are discussed in Section 3. In Section 4 we discuss how the formulations can be tightened with valid inequalities. The computational study is reported in Section 5. Conclusions and final remarks are presented in Section 6. A glossary of problem and model acronyms is given in Appendix A. 


\section{Mathematical Formulations}

In this section we introduce two distinct arc-load formulations. It is mainly the network structure that differs in the two formulations. Since a ship can visit the same port several times during the planning horizon, one needs to define the ship visits to each port unambiguously. One approach consists of adapting a discrete time formulation of the SSIRP by performing a discretization of the time to overcome the complicating factor of handling the multiple visits to each port. The other approach is to consider an ordering of the visits, and introduce an index indicating the visit number to a particular port. Hence, each network node corresponds to an event. This approach corresponds to the continuous time formulation. The first network is in general larger than the second one. However while the first network can have only cycles within each time period, the second one includes many cycles.

Continuous time formulations have normally been applied when the consumption rates are constant during the time horizon and the inventory bounds (safety stocks and upper bound capacity) are satisfied during the entire time horizon. However the discrete time formulations are normally applied when the consumption rates are varying during the time horizon, but are constant within a time period. For these models the inventory bounds are normally guaranteed at the end/beginning of each time period, only.

First, we introduce the SSIRP formulation for the time varying consumption rates problem and call it the Basic Arc-Load Discrete Time formulation with time varying consumption (BD-SSIRP-V). Then we explain the changes of the formulation for the problem with constant consumption rates and where inventory levels should be within the inventory bounds during the entire planning horizon, and call it BD-SSIRP. In both models, the time is discretized into time periods. A node in the underlying network is described by the port and time period.

The discrete time formulation presented here deviates from the discrete time formulation in [1], where a ship could stay in port for several time periods, and from [19] where each port operation was estimated to take one time period and the sailing an integer number of time periods. Here, a time period is greater or equal to the maximum time for loading/unloading, and may also include several port visits and sailings between ports. Thus, the time discretization needs to be appropriately chosen. The time unit should be simultaneously large enough to accommodate the duration of a full ship operation, and fine enough as certain constraints can only be ensured over the entire period or at the end of each time period. For example, restricting the number of operating vessels in a port can only be enforced over the entire time period, and constraints such as inventory capacity, are only enforced at the end of each time period. In addition, the consumption rate needs to be constant within a time period in the case of time varying consumption.

Demand rates and consumption rates could be used interchangeably, but we use consumption rates throughout the chapter.

An example of the ship routes in a feasible solution is depicted in Figure 1. Ship 1 sails from its origin to port 2. Then it starts to operate in period 2 at port 2. Further on, it sails to port 4 and starts to operate in period 3 at port 4 . Then it sails to port 3 and starts to operate at port 3 in period 7 . Finally the ship sails to the destination. Observe that the period that defines a visit is the period at which the ship starts to operate.

The second formulation is called the Basic Arc-Load Continuous Time formulation (BCSSIRP) and has been used by several authors when the consumption rates are constant during the planning horizon, see for instance [5] and [9]. For each port, we define a sequence of events associated with the vessel arrivals. Each event is represented by a pair: (port, order of the arrival). Ship paths are illustrated in Figure 2. For instance, ship 2 leaves origin $\mathrm{O}_{2}$ and 

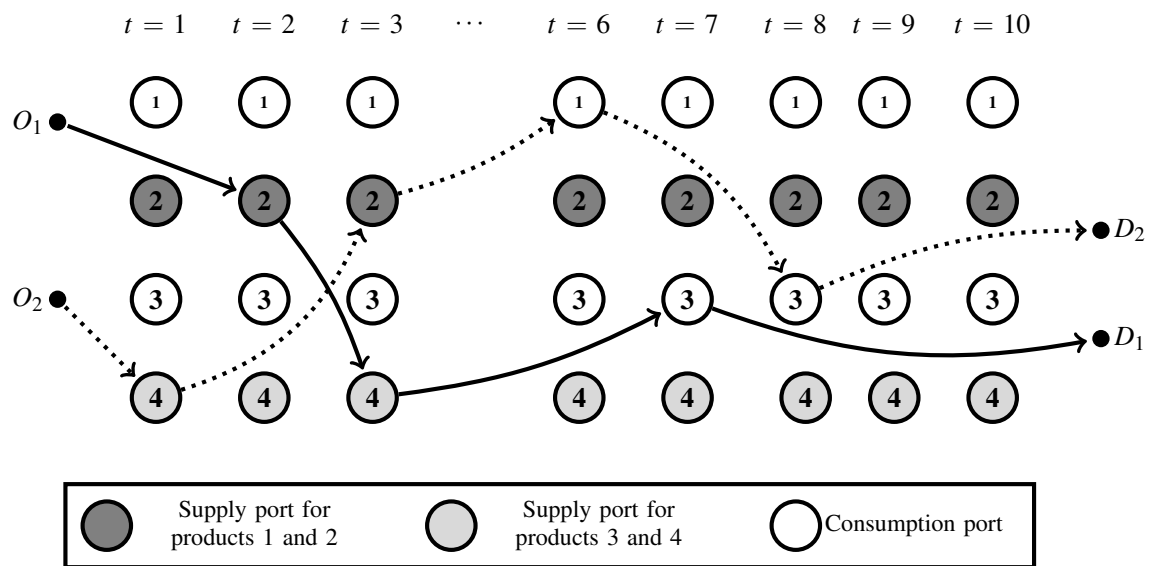

Fig. 1 Example of two routes with a discrete time network. Each column corresponds to a period and each horizontal layer corresponds to a port.

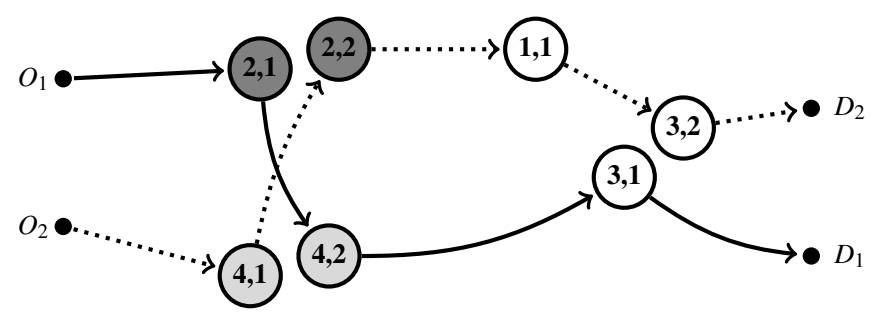

Fig. 2 Example of ship routes where each node represents a visit. The first label indicates the port and the second label indicates the visit. Each arc type represents the path of a different ship.

sails to port 4 (for the first visit to this port), then sails to port 2 (for the second visit to this port, since the first visit was made by ship 1), and sails to port 1 for its first visit. Finally, the ship sails to port 3 (for the second visit to port 3 , since the first visit was made by ship 1) before it ends at its destination.

\subsection{Arc-Load Discrete Time Formulations}

In this section we present the basic arc-load models BD-SSIRP-V and BD-SSIRP for the time varying consumption and constant consumption, respectively. The finite time horizon is divided into a discrete number of periods. A ship path is defined as a sequence of pairs (port, period) representing the nodes of the network. The period that defines a visit is the period in which the ship starts to operate. Waiting, operating and traveling times are considered in a continuous time measure.

First we introduce the model BD-SSIRP-V. Then we adapt this formulation for the constant consumption rate case. 


\subsubsection{SSIRP with time varying consumption rates}

The BD-SSIRP-V is similar to the formulation introduced in [2], but some of the problem specific details are skipped. To the best of our knowledge, the model has never been used for constant consumption rates. In this model, all variables will have a superscript $D$ to indicate the discrete time model.

The presentation of the formulation is split into the following parts: routing constraints, loading and unloading constraints, time constraints and inventory constraints. The objective function is presented at the end.

\section{Routing constraints:}

Let $V$ denote the set of ships. Each ship $v \in V$ must depart from its initial position (in the beginning of the planning horizon) that can be in a port or a point at sea. The set of ports is denoted by $N$ and the set of periods is denoted by $T$.

For the routing we define the following binary variables: $x_{i t j u v}^{D}$ is equal to 1 if ship $v$ starts to operate at port $i$ in period $t$ and then sails from port $i$ to port $j$ and starts to operate at port $j$ in period $u$; and 0 otherwise, while $x_{i t v}^{O D}$ indicates whether ship $v$ sails directly from its initial position to port $i$ to start an operation in period $t$ or not. $x_{i t v}^{O D}$ could have been included in $x_{i t j u v}^{D}$, but is introduced to ease the reading. Variable $z_{i t v}^{D}$ is 1 if ship $v$ ends its route at port $i$ after an operation that started in time period $t$; and 0 otherwise, and $z_{v}^{O D}$ is 1 if ship $v$ ends its route at the origin (it is not used) and 0 otherwise. Variable $w_{i t v}^{D}$ is 1 if ship $v$ visits port $i$ in period $t$; and 0 otherwise. Finally, $y_{i t}^{D}$ is 1 if some ship visits port $i$ in period $t ; 0$ otherwise.

Variables $x_{i t j u v}^{D}$ are not defined for $t>u$. For ease of notation we include them in the model assuming they are zero. We allow them to be positive if $t=u$, that means a ship can visit two ports in succession in the same time period. We also assume $x_{i t j u v}^{D}=0$ if $i=j$.

The routing constraints are as follows:

$$
\begin{aligned}
& \sum_{i \in N} \sum_{t \in T} x_{i t v}^{O D}+z_{v}^{O D}=1, \quad \forall v \in V, \\
& w_{i t v}^{D}-\sum_{j \in N} \sum_{u \in T} x_{j u i t v}^{D}-x_{i t v}^{O D}=0, \quad \forall v \in V, i \in N, t \in T, \\
& w_{i t v}^{D}-\sum_{j \in N} \sum_{u \in T} x_{i t j u v}^{D}-z_{i t v}^{D}=0, \quad \forall v \in V, i \in N, t \in T, \\
& \sum_{v \in V} w_{i t v}^{D}=y_{i t}^{D}, \quad \forall i \in N, t \in T, \\
& x_{i t j u v}^{D} \in\{0,1\}, \quad \forall v \in V, i, j \in N, t, u \in T, \\
& x_{i t v}^{O D}, w_{i t v}^{D}, z_{i t v}^{D} \in\{0,1\}, \quad \forall v \in V, i \in N, t \in T, \\
& y_{i t}^{D} \in\{0,1\}, \quad \forall i \in N, t \in T, \\
& z_{v}^{O D} \in\{0,1\}, \quad \forall v \in V .
\end{aligned}
$$

Constraints (1) ensure that ship $v$ either departs from its initial position to port $i$ in period $t$ or it is not used. Constraints (2) and (3) are the flow conservation constraints ensuring that a ship arriving at a port also leaves that port by either visiting another port or ending its route. Equations (4) guarantee that at most one ship can operate at port $i$ in a given time period. 
Constraints (5)-(8) define the variables as binary.

Loading and unloading:

Let $K$ represent the set of products and $K_{v}$ represent the set of products that ship $v$ can transport. Not all ports consume all products. Parameter $J_{i k}$ assumes value 1 if port $i$ is a supplier of product $k ;-1$ if port $i$ is a consumer of product $k$, and 0 if $i$ is neither a consumer nor a supplier of product $k$. The quantity of product $k$ on board ship $v$ at the beginning of the planning horizon is given by $Q_{v k}$. $C_{v k}$ is the capacity of the compartment of ship $v$ dedicated for product $k$. The minimum and maximum discharge quantities of product $k$ are given by $\underline{Q}_{i k}$ and $\bar{Q}_{i k}$, respectively.

In order to model the loading and unloading constraints we define the following binary variables: $o_{i t v k}^{D}$ is equal to 1 if product $k$ is loaded onto or unloaded from ship $v$ at port $i$ in time period $t$, and 0 otherwise; and the following continuous variables: $q_{i t v k}^{D}$ is the amount of product $k$ loaded onto or unloaded from $\operatorname{ship} v$ at port $i$ in time period $t, l_{i t v k}^{D}$ is the amount of product $k$ on board ship $v$ when leaving from port $i$ after an operation that started in time period $t$. For ease of notation, variables $o_{i t v k}^{D}$, such that $J_{i k}=0$, are included in the model and assumed to be zero.

The loading and unloading constraints are given by:

$$
\begin{aligned}
& x_{i t j u v}^{D}\left(l_{i t v k}^{D}+J_{j k} q_{j u v k}^{D}-l_{j u v k}^{D}\right)=0, \quad \forall v \in V, i, j \in N, t, u \in T, k \in K_{v}, \\
& x_{i t v}^{O D}\left(Q_{v k}+J_{i k} q_{i t v k}^{D}-l_{i t v k}^{D}\right)=0, \quad \forall v \in V, i \in N, t \in T, k \in K_{v}, \\
& l_{i t v k}^{D} \leq C_{v k} \sum_{j \in N} \sum_{u \in T} x_{i t j u v}^{D}, \quad \forall v \in V, i \in N, t \in T, k \in K_{v}, \\
& q_{i t v k}^{D} \leq C_{v k} o_{i t v k}^{D}, \quad \forall v \in V, i \in N, t \in T, k \in K_{v}: J_{i k}=1, \\
& \underline{Q}_{i k} o_{i t v k}^{D} \leq q_{i t v k}^{D} \leq \min \left\{C_{v k}, \bar{Q}_{i k}\right\} o_{i t v k}^{D}, \quad \forall v \in V, i \in N, t \in T, k \in K_{v}: J_{i k}=-1, \\
& \sum_{k \in K_{v}} o_{i t v k}^{D} \geq w_{i t v}^{D}, \quad \forall v \in V, i \in N, t \in T, \\
& o_{i t v k}^{D} \leq w_{i t v}^{D}, \quad \forall v \in V, i \in N, t \in T, k \in K_{v}, \\
& l_{i t v k}^{D}, q_{i t v k}^{D} \geq 0, \quad \forall v \in V, i \in N, t \in T, k \in K_{v}, \\
& o_{i t v k}^{D} \in\{0,1\}, \quad \forall v \in V, i \in N, t \in T, k \in K_{v} .
\end{aligned}
$$

Constraints (9) and (10) relate the quantity on board to the quantity loaded and/or unloaded. Constraints (9) ensure that if ship $v$ sails from port $i$ (after an operation started in period $t$ ) to port $j$ (to initialize an operation in period $u$ ), then the quantity of product $k$ on board at the departure from island $j$ should be equal to the quantity on board at departure from port $i$ plus (resp. minus) the quantity loaded (resp. unloaded) from $j$. Equations (10) relate the quantity on board with the quantity loaded and/or unloaded in the starting position. Constraints (11) impose an upper bound on the quantity on board. They also ensure that if the quantity on board is positive than the ship must travel to some other port. Constraints (12) ensure that if an operation occurs at a loading port, that is, $q_{i t v k}^{D}>0$, than the setup variable $o_{i t v k}^{D}$ must be one. They also impose an upper bound on the quantity loaded. Constraints (13) impose lower and upper limits on the unload quantities, respectively. Constraints (14) ensure that if ship $v$ starts an operation at port $i$ in time period $t$, then at least one product must be (un)loaded. Constraints (15) ensure that if ship $v$ (un)loads one product at port $i$ in period $t$, then $w_{i t v}^{D}$ must be one. The nonnegativity requirements (16) are given for the vari- 
ables representing the load on board and the (un)loading quantity. Finally, the formulation involves binary requirements (17) on the operating variables.

Constraints (9) and (10) are non-linear. Following [15], equations (9) can be linearized by replacing them with the following two sets of constraints:

$$
\begin{array}{cc}
l_{i t v k}^{D}+J_{j k} q_{j u v k}^{D}-l_{j u v k}^{D}+C_{v k} x_{i t j u v}^{D} \leq C_{v k}, & \forall v \in V, i, j \in N, t, u \in T, k \in K_{v}, \\
l_{i t v k}^{D}+J_{j k} q_{j u v k}^{D}-l_{j u v k}^{D}-C_{v k} x_{i t j u v}^{D} \geq-C_{v k}, & \forall v \in V, i, j \in N, t, u \in T, k \in K_{v},
\end{array}
$$

and equations (10) can be replaced by:

$$
\begin{aligned}
& Q_{v k}+J_{i k} q_{i t v k}^{D}-l_{i t v k}^{D}+C_{v k} x_{i t v}^{O D} \leq C_{v k}, \\
& Q_{v k}+J_{i k} q_{i t v k}^{D}-l_{i t v k}^{D}-C_{v k} x_{i t v}^{O D} \geq-C_{v k}, \quad \forall v \in V, i \in N, t \in T, k \in K_{v},
\end{aligned}
$$

Time constraints:

We have chosen a discrete time formulation consisting of few time periods compared to most existing discrete time formulations in the literature, see e.g. [1] and [16]. In order to account for the time aspects correctly we consider a continuous time measure in addition to the discrete time. In comparison to other discrete time MIRP formulations [1], we do not need to explicitly define binary waiting variables and by this avoid the symmetry problem that many such models have.

We define the following parameters: $T_{i k}^{Q}$ is the time required to load/unload one unit of product $k$ at port $i$; $T_{i k}^{S}$ is the set up time required to operate product $k$ at port $i$. Parameter $T_{i j v}$ is the traveling time between port $i$ and $j$ by ship $v ; T_{i v}^{O}$ indicates the traveling time required by ship $v$ to sail from its initial port position to port $i ; T_{i}^{B}$ is the minimum interval between the departure of one ship and the next arrival at port $i$. Finally, $\bar{T}$ is the length of the time horizon.

We define the nonnegative continuous variables $t_{i t}^{D}$ as the start time of the operation at port $i$ in time period $t$, and $t_{i t}^{E D}$ as the end time of the operation that started during period $t$ in port $i$. The time constraints are as follows,

$$
\begin{aligned}
& t_{i t}^{E D} \geq t_{i t}^{D}+\sum_{v \in V} \sum_{k \in K_{v}} T_{i k}^{S} o_{i t v k}^{D}+\sum_{v \in V} \sum_{k \in K_{v}} T_{i k}^{Q} q_{i t v k}^{D}, \quad \forall i \in N, t \in T, \\
& t_{i t}^{D}-t_{i(t-1)}^{E D} \geq T_{i}^{B} y_{i t}^{D}, \quad \forall i \in N, t \in T: t>1, \\
& t_{i t}^{E D}+T_{i j v}-t_{j u}^{D} \leq \bar{T}\left(1-x_{i t j u v}^{D}\right), \quad \forall v \in V, i, j \in N, t, u \in T, \\
& \sum_{v \in V} T_{i v}^{O} x_{i t v}^{O D} \leq t_{i t}^{D}, \quad \forall i \in N, t \in T \\
& t-1 \leq t_{i t}^{D} \leq t, \quad \forall i \in N, t \in T \\
& t_{i t}^{D}, t_{i t}^{E D} \geq 0, \quad \forall i \in N, t \in T .
\end{aligned}
$$

Equations (22) define the end time of each operation. Notice the end time can be greater than the starting time plus the set up times and the time for the (un)load operations. This accounts the possibility of a ship to wait between (un)loadings. Constraints (23) impose a minimum interval between two consecutive visits at port $i$. Constraints (24) ensure that if $\operatorname{ship} v$ sails from port $i$ (after an operation started in period $t$ ) to port $j$ (to initialize an operation in period $u$ ), then the operation at port $j$ can only start after the end time of operation at port $i$ plus the time required to travel from $i$ to $j$. Constraints (25) ensure that if ship $v$ travels 
from its initial position to port $i$ to start an operation in period $t$, then the starting time at port $i$ can only occur after the traveling time. Constraints (26) link the continuous with the discrete time measures and constraints (27) define the sign of the continuous time variables.

When time windows are considered they can be easily included in the model. For instance, if the start of an operation at port $i$ in period $t$ is restricted to a time window $\left[A_{i t}, B_{i t}\right]$, then it suffices to replace constraints (26) by $A_{i t} \leq t_{i t}^{D} \leq B_{i t}$.

\section{Inventory constraints:}

Inventory constraints are considered for each unloading port $i\left(J_{i k}=-1\right)$. $D_{i t k}$ indicates the demand or consumption of product $k$ at port $i$ in period $t$. For each product $k$ at a consumption port $i$, the minimum stock level is given by $\underline{S}_{i k}$ and the maximum stock level (tank capacity) is given by $\bar{S}_{i k}$. $S_{i k}^{O}$ denotes the initial stock level of product $k$ in port $i$.

The nonnegative continuous variables $s_{i t k}^{D}$ indicate the stock level of product $k$ in port $i$ at the end of period $t$. The inventory constraints are as follows:

$$
\begin{aligned}
& s_{i(t-1) k}^{D}+\sum_{v \in V} q_{i t v k}^{D}-s_{i t k}^{D}=D_{i t k}, \quad \forall i \in N, t \in T, k \in K: J_{i k}=-1, \\
& s_{i 0 k}^{D}=S_{i k}^{O}, \quad \forall i \in N, k \in K: J_{i k}=-1, \\
& \underline{S}_{i k} \leq s_{i t k}^{D} \leq \bar{S}_{i k}, \quad \forall i \in N, t \in T, k \in K: J_{i k}=-1 .
\end{aligned}
$$

Constraints (28) are the inventory balance constraints. These constraints together with the bounds ensure that the demand for each product at each port in each time period is satisfied. Constraints (29) define the initial stock levels. The upper and lower bounds on the stock levels are ensured by constraints (30).

\section{Objective Function:}

The objective function is to minimize the costs (transportation and setup costs):

$$
\operatorname{Min} \sum_{v \in V} \sum_{i, j \in N} \sum_{t, u \in T} C_{i j v}^{T} x_{i t j u v}^{D}+\sum_{v \in V} \sum_{i \in N} \sum_{t \in T} C_{i v}^{T O} x_{i t v}^{O D}+\sum_{v \in V} \sum_{i \in N} \sum_{t \in T} \sum_{k \in K_{v}} C_{i k}^{O} o_{i t v k}^{D}
$$

where $C_{i j v}^{T}$ is the total transportation cost for ship $v$ to sail from port $i$ to port $j, C_{i v}^{T O}$ is the cost for ship $v$ to sail from its origin to port $i$, and $C_{i k}^{O}$ is the fixed cost of operating (load/unload) product $k$ at port $i$.

The basic arc-load discrete time formulation with time varying consumption rates, BDSSIRP-V, is given by (1)-(8), (11)-(31). Even though the model includes discrete time and continuous time variables we call it a discrete time formulation.

\subsubsection{SSIRP with constant consumption rates}

In this section we consider the variant of the SSIRP where constant consumption rates are assumed. The two related problems occur in two different planning problems. The time varying consumption problem occurs when a set of orders are given. Each order corresponds 


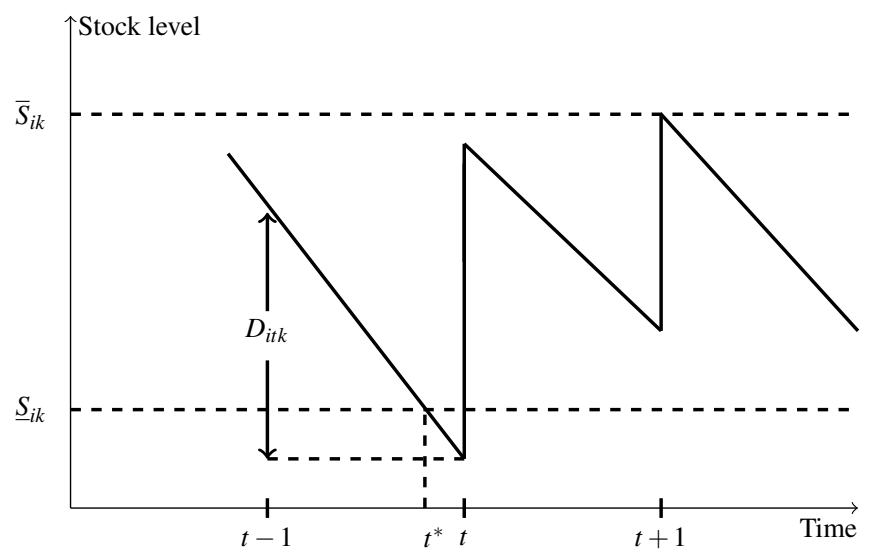

Fig. 3 Delivery must occur no later than $t^{*}$ in SSIRP, and it must occur no later than $t$ in the SSIRP-V.

to a quantity of an oil product that must be delivered into a specific port and has a deadline to be satisfied. The constant consumption rate is normally assumed when the planners are considering longer time horizons. In this case the consumption rates correspond to the estimated consumption rates from real data. In order to model the constant rate case we can adapt the discrete time formulation.

In the BD-SSIRP-V the safety stock is guaranteed at the end of each period only. These ends of periods are artificially established. Hence, by choosing a different discretization the model will guarantee the stock level at different times. As depicted in Figure 3 it may happen that the stock level goes below the minimum stock level in the middle of a period. This situation should not be allowed in the constant rate case, SSIRP, where the safety stock must be satisfied at any time in the interval $[0, \bar{T}]$.

In order to prevent such a situation to occur, while keeping a chosen discretization, we add the following constraints

$$
s_{i(t-1) k}^{D}-D_{i t k}\left(t_{i t}^{D}-t+1\right) \geq \underline{S}_{i k}, \quad \forall i \in N, t \in T, k \in K: J_{i k}=-1 .
$$

The left hand side of (32) measures the stock level at the start time of the operation which is the stock level at the beginning of the period minus the consumption until the start of the operation. These levels should be above the safety stock levels.

Similarly, in order to prevent stock to go above the tank capacity at the end of a discharge operation, we add the following constraints

$$
s_{i(t-1) k}^{D}-D_{i t k}\left(t_{i t}^{E D}-t+1\right)+\sum_{v \in V} q_{i t v k}^{D} \leq \bar{S}_{i k}, \quad \forall i \in N, t \in T, k \in K: J_{i k}=-1 .
$$

Constraints (32) and (33) could also have been added to BD-SSIRP-V if it is important to ensure that the inventory levels are within the limits during the time horizon.

The basic arc-load discrete time formulation with constant consumption rates, BDSSIRP, is given by (1)-(8), (11)- (33). 


\subsection{Arc-Load Continuous Time Formulation}

In this section we present the basic arc-load continuous time formulation, BC-SSIRP, for the case with constant consumption rates.

In the BD-SSIRP we discretized the time such that in each period at most one visit could occur at each port. Here, we present an alternative formulation where port events, here called port visits, are distinguished by the order of the visit. This type of formulation was used in [5] and [9].

Again, we divide the set of constraints of the formulation into the following parts: routing constraints, loading and unloading constraints, time constraints and inventory constraints.

Contrary to the discrete model that combines both discrete and continuous time, this model uses continuous time only.

For each port we consider an ordering of the visits according to the time of the visit. The ship paths are defined on a network where the nodes are represented by a pair $(i, m)$, where $i$ is the port and $m$ is the visit number at port $i$.

For this formulation only the new notation is introduced.

\section{Routing constraints:}

Each possible port visit is denoted by the pair $(i, m)$ representing the $m^{\text {th }}$ visit to port $i$. Direct ship movements (arcs) from port visit $(i, m)$ to port visit $(j, n)$ are represented by $(i, m, j, n)$.

We define $S^{A}$ as the set of possible port visits $(i, m), S_{v}^{A}$ as the set of possible port visits made by ship $v$, and set $S_{v}^{X}$ as the set of all possible movements $(i, m, j, n)$ of ship $v$.

For the routing we define the following binary variables: $x_{i m j n v}^{C}$ is equal to 1 if ship $v$ sails from port visit $(i, m)$ directly to port visit $(j, n)$; and 0 otherwise, $x_{i m v}^{O C}$ indicates whether ship $v$ sails directly from its initial position to port visit $(i, m)$ or not, $w_{i m v}^{C}$ is 1 if ship $v$ visits port $i$ at arrival $(i, m)$; and 0 otherwise, $z_{i m v}^{C}$ is equal to 1 if ship $v$ ends its route at port visit $(i, m)$; and 0 otherwise, and $y_{i m}^{C}$ indicates whether a ship is visiting port arrival $(i, m)$ or not.

The routing constraints are as follows:

$$
\begin{aligned}
& \sum_{(i, m) \in S_{v}^{A}} x_{i m v}^{O C}+z_{v}^{O C}=1, \quad \forall v \in V, \\
& w_{i m v}^{C}-\sum_{(j, n) \in S_{v}^{A}} x_{j n i m v}^{C}-x_{i m v}^{O C}=0, \quad \forall v \in V,(i, m) \in S_{v}^{A}, \\
& w_{i m v}^{C}-\sum_{(j, n) \in S_{v}^{A}} x_{i m j n v}^{C}-z_{i m v}^{C}=0, \quad \forall v \in V,(i, m) \in S_{v}^{A}, \\
& \sum_{v \in V} w_{i m v}^{C}=y_{i m}^{C}, \quad \forall(i, m) \in S^{A}, \\
& y_{i(m-1)}^{C}-y_{i m}^{C} \geq 0, \quad \forall(i, m) \in S^{A}: m>1, \\
& x_{i m v}^{O C}, w_{i m v}^{C}, z_{i m v}^{C} \in\{0,1\}, \quad \forall v \in V,(i, m) \in S_{v}^{A}, \\
& x_{i m j n v}^{C} \in\{0,1\}, \quad \forall v \in V,(i, m, j, n) \in S_{v}^{X}, \\
& y_{i m}^{C} \in\{0,1\}, \quad \forall(i, m) \in S^{A}, \\
& z_{v}^{O C} \in\{0,1\}, \quad \forall v \in V .
\end{aligned}
$$


Equations (34) ensure that each ship departs from its initial position and sails towards another port or the ship is not used. Equations (35) and (36) are the flow conservation constraints, ensuring that a ship arriving at a port also leaves that port by either visiting another port or ending its route. Constraints (37) ensure that each port visit $(i, m)$ is made at most once. Constraints (38) state that if port $i$ is visited $m$ times, then it must also have been visited $m-1$ times. Constraints (39)-(42) define the variables as binary.

\section{Loading and unloading:}

In order to model the loading and unloading constraints, we define the following binary variables: $o_{i m v k}^{C}$ is equal to 1 if product $k$ is loaded onto or unloaded from ship $v$ at port visit $(i, m)$, and 0 otherwise. In addition, we define the following continuous variables: $q_{i m v k}^{C}$ is the amount of product $k$ (un)loaded at port visit $(i, m)$ and $l_{i m v k}^{C}$ is the amount of product $k$ on board ship $v$ when leaving from port visit $(i, m)$.

The loading and unloading constraints are given by:

$$
\begin{aligned}
& x_{i m j n v}^{C}\left(l_{i m v k}^{C}+J_{j k} q_{j n v k}^{C}-l_{j n v k}^{C}\right)=0, \quad \forall v \in V,(i, m, j, n) \in S_{v}^{X}, k \in K_{v}, \\
& x_{i m v}^{O C}\left(Q_{v k}+J_{i k} q_{i m v k}^{C}-l_{i m v k}^{C}\right)=0, \quad \forall v \in V,(i, m) \in S_{v}^{A}, k \in K_{v}, \\
& l_{i m v k}^{C} \leq C_{v k} \sum_{(j, n) \in S_{v}^{A}} x_{i m j n v}^{C}, \quad \forall v \in V,(i, m) \in S_{v}^{A}, k \in K_{v}, \\
& q_{i m v k}^{C} \leq C_{v k} o_{i m v k}^{C}, \quad \forall v \in V,(i, m) \in S_{v}^{A}, k \in K_{v}: J_{i k}=1, \\
& \underline{Q}_{i k} o_{i m v k}^{C} \leq q_{i m v k}^{C} \leq \min \left\{C_{v k}, \bar{Q}_{i k}\right\} o_{i m v k}^{C}, \quad \forall v \in V,(i, m) \in S_{v}^{A}, k \in K_{v}: J_{i k}=-1, \\
& \sum_{k \in K_{v}} o_{i m v k}^{C} \geq w_{i m v}^{C}, \quad \forall v \in V,(i, m) \in S_{v}^{A}, \\
& o_{i m v k}^{C} \leq w_{i m v}^{C}, \quad \forall v \in V,(i, m) \in S_{v}^{A}, k \in K_{v}, \\
& l_{i m v k}^{C}, q_{i m v k}^{C} \geq 0, \quad \forall v \in V,(i, m) \in S_{v}^{A}, k \in K_{v}, \\
& o_{i m v k}^{C} \in\{0,1\}, \quad \forall v \in V,(i, m) \in S_{v}^{A}, k \in K_{v} .
\end{aligned}
$$

Equations (43) determine the quantity of product $k$ on board ship $v$ when the ship sails from port visit $(i, m)$ to port visit $(j, n)$. These constraints can be linearized as follows:

$$
\begin{aligned}
& l_{i m v k}^{C}+J_{j k} q_{j n v k}^{C}-l_{j n v k}^{C}+C_{v k} x_{i m j n v}^{C} \leq C_{v k}, \quad \forall v \in V,(i, m, j, n) \in S_{v}^{X}, k \in K_{v}, \\
& l_{i m v k}^{C}+J_{j k} q_{j n v k}^{C}-l_{j n v k}^{C}-C_{v k} x_{i m j n v}^{C} \geq-C_{v k}, \quad \forall v \in V,(i, m, j, n) \in S_{v}^{X}, k \in K_{v} .
\end{aligned}
$$

Constraints (44) are similar to (43) and determine the load on board the ship for the first ship visit. These constraints can be replaced by the following linear constraints:

$$
\begin{array}{lr}
Q_{v k}+J_{i k} q_{i m v k}^{C}-l_{i m v k}^{C}+C_{v k} x_{i m v}^{O C} \leq C_{v k}, & \forall v \in V,(i, m) \in S_{v}^{A}, k \in K_{v}, \\
Q_{v k}+J_{i k} q_{i m v k}^{C}-l_{i m v k}^{C}-C_{v k} x_{i m v}^{O C} \geq-C_{v k}, & \forall v \in V,(i, m) \in S_{v}^{A}, k \in K_{v} .
\end{array}
$$

The ship capacity constraints are given by (45). Constraints (46) impose an upper bound on the quantity loaded at the supply port. Constraints (47) impose lower and upper limits on the unload quantities. Constraints (48) ensure that if ship $v$ makes port visit $(i, m)$, then at least one product must be (un)loaded. Constraints (49) ensure that if ship $v$ (un)loads one product at visit $(i, m)$, then $w_{i m v}^{C}$ must be one. Constraints (50)-(51) are the non-negativity and integrality constraints. 


\section{Time constraints:}

Given the start time and end time variables, $t_{i m}^{C}$ and $t_{i m}^{E C}$ at port visit $(i, m)$, the time constraints can be written as:

$$
\begin{aligned}
& t_{i m}^{E C} \geq t_{i m}^{C}+\sum_{v \in V} \sum_{k \in K_{v}} T_{i k}^{Q} q_{i m v k}^{C}+\sum_{v \in V} \sum_{k \in K_{v}} T_{i k}^{S} o_{i m v k}^{C}, \quad \forall(i, m) \in S^{A}, \\
& t_{i m}^{C}-t_{i(m-1)}^{E C}-T_{i}^{B} y_{i m}^{C} \geq 0, \quad \forall(i, m) \in S^{A}: m>1, \\
& t_{i m}^{E C}+T_{i j v}-t_{j n}^{C} \leq \bar{T}\left(1-x_{i m j n v}^{C}\right), \quad \forall v \in V,(i, m, j, n) \in S_{v}^{X}, \\
& \sum_{v \in V} T_{i v}^{O} x_{i m v}^{O C} \leq t_{i m}^{C}, \quad \forall(i, m) \in S^{A}, \\
& t_{i m}^{C}, t_{i m}^{E C} \geq 0, \quad \forall(i, m) \in S^{A} .
\end{aligned}
$$

Constraints (56) define the end time of service of port visit $(i, m)$. Constraints (57) impose a minimum interval between two consecutive visits at port $i$. Constraints (58) relate the end time of port visit $(i, m)$ to the start time of port visit $(j, n)$ when ship $v$ sails directly from port $(i, m)$ to $(j, n)$. Constraints (59) ensure that if ship $v$ travels from its initial position directly to port visit $(i, m)$, then the start time is at least the traveling time between the two positions. Constraints (60) define the continuous time variables.

Single time windows for each visit can be introduced in a similar way as for the discrete case. However in case the time windows are associated with open hours at ports then new variables are necessary to model multiple time windows.

\section{Inventory constraints:}

The inventory constraints are necessary to ensure that the inventory levels are kept within the corresponding bounds and to link the inventory levels to the (un)loading quantities.

We define parameter $R_{i k}$ as the consumption rate of product $k$ at port $i$ (that is, $D_{i t k}=$ $\left.R_{i k}, \forall t \in T\right)$, and define the nonnegative continuous variables $s_{i m k}^{C}$ and $s_{i m k}^{E C}$ indicating the inventory levels at the start and at the end of port visit $(i, m)$, respectively. The inventory constraints are as follow:

$$
\begin{aligned}
& s_{i 1 k}^{C}=S_{i k}^{O}-R_{i k} t_{i 1}^{C}, \quad \forall i \in N, k \in K: J_{i k}=-1, \\
& s_{i m k}^{E C}=s_{i m k}^{C}+\sum_{v \in V} q_{i m v k}^{C}-R_{i k}\left(t_{i m}^{E C}-t_{i m}^{C}\right), \quad \forall(i, m) \in S^{A}, k \in K: J_{i k}=-1, \\
& s_{i m k}^{C}=s_{i(m-1) k}^{E C}-R_{i k}\left(t_{i m}^{C}-t_{i(m-1)}^{E C}\right), \quad \forall(i, m) \in S^{A}: m>1, k \in K: J_{i k}=-1, \\
& \underline{S}_{i k} \leq s_{i m k}^{C}, s_{i m k}^{E C} \leq \bar{S}_{i k}, \quad \forall(i, m) \in S^{A}, k \in K: J_{i k}=-1 .
\end{aligned}
$$

Equations (61) calculate the inventory level of each product at the first visit. Equations (62) calculate the inventory level of each product when the service ends at port visit $(i, m)$. Similarly, equations (63) relate the inventory level at the start of port visit $(i, m)$ to the inventory level at the end of port visit $(i, m-1)$. The upper and lower bounds on the inventory levels are ensured by constraints (64). 
It remains to ensure that the inventory levels at the end of the planning horizon is within the inventory limits. We discuss two options. The following set of constraints was used in [22].

$$
\underline{S}_{i k} \leq s_{i m k}^{E C}-R_{i k}\left(\bar{T}-t_{i m}^{E C}\right)\left(y_{i m}^{C}-y_{i(m+1)}^{C}\right) \leq \bar{S}_{i k}, \quad \forall(i, m+1) \in S^{A}, k \in K: J_{i k}=-1 .
$$

We can see that $t_{i m}^{E C}$ is the end time of the last visit to port $i$ if and only if $y_{i m}^{C}-y_{i(m+1)}^{C}=1$. This set of constraints is nonlinear and can be linearized as in [22]. However we omit the linearization process here, because we will follow the approach used in [9], to handle the stock level at the end of the planning horizon. Consider the following set of constraints where $\bar{\mu}_{i}$ is an upper bound on the number of visits to port $i$.

$$
\underline{S}_{i k} \leq s_{i \bar{\mu}_{i} k}^{E C}+R_{i k}\left(\bar{T}-t_{i \bar{\mu}_{i}}^{E C}\right) \leq \bar{S}_{i k}, \quad \forall i \in N, k \in K: J_{i k}=-1,
$$

Here the end time of the last possible visit is given by $t_{i \bar{\mu}_{i}}^{E C}$.

\section{Objective function:}

The objective is to minimize the total routing and operating cost:

$$
\sum_{v \in V} \sum_{(i, m, j, n) \in S_{v}^{X}} C_{i j v}^{T} x_{i m j n v}^{C}+\sum_{v \in V} \sum_{(i, m) \in S_{v}^{A}} C_{i v}^{T O} x_{i m v}^{O C}+\sum_{v \in V} \sum_{(i, m) \in S_{v}^{A}} \sum_{k \in K_{v}} C_{i k}^{O} o_{i m v k}^{C}
$$

The basic arc-load time continuous formulation with constant consumption rates, BCSSIRP, is defined by (34)-(42), (45)-(66).

\subsection{Comparison of the Discrete Time and Continuous Time Models}

Here we discuss the two models regarding their integrality gaps, size, and level of information provided.

\section{Integrality gaps}

Although the definition of the variables in the time discrete model is different from the definition of variables in the time continuous model, we can easily see that the two mathematical models are very similar. In fact, removing the inventory constraints from both models and constraints (38) from the BC-SSIRP, the mathematical expressions of both models is similar. The unique difference is that variables $x_{i t j u v}^{D}$ are defined for all $u \geq t$ while $x_{i m j n v}^{C}$ are defined for all $m$ and $n$. As a consequence the linear relaxation of the discrete model BD-SSIRP without inventory constraints should provide bounds at least as good as those provided by the linear relaxation of the continuous model BC-SSIRP without inventory constraints.

To compare theoretically the complete models (with inventory variables) is not a straightforward task since one needs to relate the two sets of variables. Here we only provide an experimental comparison. This study is conducted in Section 5 and shows that the bounds provided by the two models are the same for the tested instances, which reinforces our comment on the similarity of the models. The computational study also shows that the integrality gaps of BD-SSIRP and BC-SSIRP are very large. In the two following sections we improve these formulations by deriving tighter extended formulations (Section 3 ) and by including valid inequalities (Section 4). The ideas used in those improvements are similar for both types of formulations. 
Size of the models

The size of the models is determined by the number of $x$ (routing) variables since this number establishes the bound for the number of variables and constraints. Contrary to the discrete model, where the number of routing variables is well defined for a particular discretization, in the continuous case this number depends on the maximum number of visits to each port $i, \bar{\mu}_{i}$. These upper bounds $\bar{\mu}_{i}$ can be computed using the minimum (un)loading quantities $Q_{i}^{k}$ and the time constraints. However, usually the quantities $Q_{i}^{k}$ are not imposed by any real limit but to avoid a "large" number of visits. Our experience showed that the maximum number of visits can be set to a minimum number of visits (computed in Section 4) plus a constant: one, two or three, depending on the port activity. For larger increases of $\bar{\mu}_{i}$, only the running time increases, see Section 5 .

We can also eliminate some routing variables $x_{i t j u v}^{D}$ from the discrete model. Since the maximum distance between two ports is short in the underlying real short sea inventory routing problem, we can eliminate variables where $u>>t$. In Section 5, we present computational experiments to evaluate the impact of the objective function, the size of the model, and on the running time of these restrictions on the variables.

\section{Information provided}

The solution of each model provides different information. However, the solution from one model can easily be converted into a solution of the other. In the discrete formulation, the information of the period in which the visits occur is given by the time variables $t_{i t}^{D}$ as well as the routing variables $x_{i t j u v}^{D}$, while in the continuous model this information is provided only by the time variables. This difference allows us to relate the routing aspects directly to the inventory in the discrete models. As we will see in Section 4, this property can be used to tighten the discrete model.

\section{Linear Relaxations and Extended Formulations}

In this section we discuss some of the weaknesses of the arc-load formulations and introduce two extended formulations for each type of model (discrete time and continuous time). We consider only the SSIRP with constant consumption rates.

In Figure 4 we present a fractional solution of the arc-load continuous time model that illustrates the weaknesses of the arc-load formulations.

As we can see from the example, the fractional solution does not guarantee the equilibrium of the flow on board the ship. Both ships unload products that they do not transport. For instance, ship 2 unloads 50 units at port 1 and these units are never loaded. Next we justify how such solutions can occur. First notice that the unique link between the load on board the ship and the path of the ship is established at the nodes. Additionally, the link is established through constraints (18)-(21) in BD-SSIRP and through constraints (52)(55) in the BC-SSIRP. These linking constraints are known to be very weak. It is therefore possible to get, in a linear fractional solution, an unload operation when the ship has nothing on board. Consider the BC-SSIRP case, and suppose $J_{j k}=-1$. If $0<x_{i m j n v}^{C}<1$ and $l_{i m v k}^{C}=l_{j n v k}^{C}=0$, then the unload quantity $q_{j n v k}^{C}$ of product $k$ can be positive. More specifically $0 \leq q_{j n v k}^{C} \leq C_{v k} \min \left\{x_{i m j n v}^{C}, 1-x_{i m j n v}^{C}\right\}$.

Also, as expected, each ship follows multiple fractional paths. 


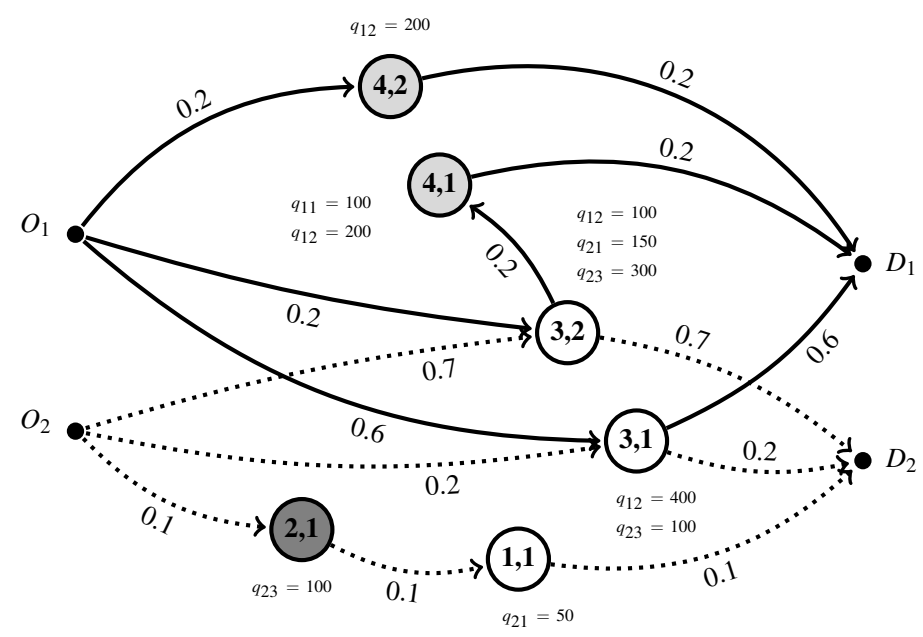

Fig. 4 Example of an optimal solution of the linear relaxation of the BC-SSIRP. The quantities $q_{v k}$ next to node $(i, m)$ represent the quantity of product $k$ unloaded by ship $v$ in the $m^{\text {th }}$ visit to port $i$. In this solution there are no loadings. The arc labels represent the values of the corresponding arc-variables. Dark arcs represent ship 1 and dashed arcs represent ship 2. We assume $Q_{v k}=0, \forall v \in V, k \in K_{v}$.

In order to avoid some of the drawbacks of the arc-load formulations, we propose two extended formulations for each approach. The new set of variables introduced in each formulation provides additional information about the solution. That information will be essential to derive tighter models. All the formulations presented in the chapter are compact. In general, the linear relations of the extended formulations lead to better bounds but are harder (considering the computational effort) to obtain. When using such formulations in a branch and bound scheme, the number of tree nodes tends to be less than in the case where a smaller formulation is used. However, the time spent in each node is usually greater.

In the first extended formulation, new variables indicating the amount of each product carried along an arc are introduced. These new variables can be seen as defining the flow of individual products along the chosen paths resulting from the routing variables for each ship. The second extended formulation can be seen as a classical multi-commodity reformulation of the first extended formulation where the flow variables additionally indicate the destination of each product along the chosen paths.

\subsection{Arc-Load Flow Reformulations}

In this section we introduce new arc-load flow variables that indicate the amount of each product carried along each arc. These flow variables allow us to assign a flow of each product to the ship path. In this way we can prevent fractional solutions as the one depicted in Figure 4.

\subsubsection{Discrete time reformulation}

Next, we present the arc-load flow discrete time formulation with constant consumption rates (FD-SSIRP). Let us define $f_{i t j u v k}^{D}$, as the amount of product $k$ that ship $v$ transports 
from port $i$, after an operation that started in period $t$, to port $j$ in order to start an operation in period $u$. For ease of notation, when $x_{i t j u v}^{D}=0$, variables $f_{i t j u v k}^{D}$ are included in the model and set to zero.

Let $f_{i t v k}^{O D}$ denote the amount of product $k$ that ship $v$ transports from its initial port position to port $i$ in period $t$.

The two sets of variables $l_{i t v k}^{D}$ and $f_{i t j u v k}^{D}$ can be related using the following equations

$$
l_{i t v k}^{D}=\sum_{j \in N} \sum_{u \in T} f_{i t j u v k}^{D}, \quad \forall v \in V, i \in N, t \in T, k \in K_{v},
$$

Constrains (9), (10) and (11) can be replaced by constraints

$$
\begin{aligned}
& f_{j u v k}^{O D}+\sum_{i \in N} \sum_{t \in T} f_{i t j u v k}^{D}+J_{j k} q_{j u v k}^{D}=\sum_{i \in N} \sum_{t \in T} f_{j u i t v k}^{D}, \quad \forall v \in V, j \in N, u \in T, k \in K_{v}, \\
& f_{i t v k}^{O D}=Q_{v k} x_{i t v}^{O D}, \quad \forall v \in V, i \in N, t \in T, k \in K_{v}, \\
& f_{i t j u v k}^{D} \leq C_{v k} x_{i t j u v}^{D}, \quad \forall v \in V, i, j \in N, t, u \in T, k \in K_{v}, \\
& f_{i t j u v k}^{D} \geq 0, \quad \forall v \in V, i, j \in N, t, u \in T, k \in K_{v} .
\end{aligned}
$$

The flow conservation constraints are given by equations (68). Equations (69) determine the amount of product $k$ on board ship $v$ at departure from the initial position. Constraints (70) are the variable upper bound constraints. They relate the flow variable $f_{i t j u v k}^{D}$ to the routing variables $x_{i t j u v}^{D}$ and, together with the nonnegativity constraints (71) impose bounds on the flow variables.

The FD-SSIRP formulation is defined by (1)-(8), (12)-(17), (22)-(33), (68)-(71).

Adding constraints (70) for $j$ and $u$ we obtain

$$
\sum_{j \in N} \sum_{u \in T} f_{i t j u v k}^{D} \leq C_{v k} \sum_{j \in N} \sum_{u \in T} x_{i t j u v}^{D}
$$

Using (67) we obtain (11). Hence constraints (11) can be obtained by aggregating constraints (70). Thus, the linear relaxation of FD-SSIRP should provide better bounds than the linear relaxation of BD-SSIRP. The drawback of this model is that it increases the size by adding a large number of continuous variables and constraints.

Notice that with the inclusion of variables $f_{i t j u v k}^{D}$, variables $q_{j u v k}^{D}$ can be eliminated from the model using equations (68), that is, setting

$$
q_{j u v k}^{D}=J_{j k}\left(\sum_{i \in N} \sum_{t \in T} f_{j u i t v k}^{D}-\sum_{i \in N} \sum_{t \in T} f_{i t j u v k}^{D}-f_{j u v k}^{O D}\right), \quad \forall v \in V, j \in N, u \in T, k \in K_{v} .
$$

\subsubsection{Continuous time reformulation}

Here we define a similar flow model for the continuous time formulation, denoted by FC-SSIRP. Let $f_{i m j n v k}^{C}$ denote the amount of product $k$ that ship $v$ transports from port visit $(i, m)$ to port visit $(j, n)$ and $f_{j n v k}^{O C}$ as the amount of product $k$ that ship $v$ transports from its initial position to port visit $(j, n)$. 
Using these additional variables, constraints (43)-(45) can be replaced by the following set of constraints:

$$
\begin{aligned}
& f_{j n v k}^{O C}+\sum_{(i, m) \in S_{v}^{A}} f_{i m j n v k}^{C}+J_{j k} q_{j n v k}^{C}=\sum_{(i, m) \in S_{v}^{A}} f_{j n i m v k}^{C}, \quad \forall v \in V,(j, n) \in S_{v}^{A}, k \in K_{v}, \\
& f_{\text {imvk }}^{O C}=Q_{v k} x_{i m v}^{O C}, \quad \forall v \in V,(i, m) \in S_{v}^{A}, k \in K_{v}, \\
& f_{\text {imjnvk }}^{C} \leq C_{v k} x_{i m j n v}^{C}, \quad \forall v \in V,(i, m, j, n) \in S_{v}^{X}, k \in K_{v}, \\
& f_{\text {imjnvk }}^{C} \geq 0, \quad \forall v \in V,(i, m, j, n) \in S_{v}^{X}, k \in K_{v} .
\end{aligned}
$$

Constraints (73) ensure the equilibrium of product $k$ on board ship $v$. Equations (74) determine the quantity on board when ship $v$ sails from its initial port position to port visit $(i, m)$. Constraints (75) link the new flow variables to the arc variables and impose an upper bound on the capacity of the compartment of ship $v$ dedicated to carry product $k$.

The arc-load flow continuous time formulation with constant consumption rates, FCSSIRP, is defined by (34)-(42), (46)-(51), (56)-(66), (73)-(76).

Similar to the discrete case, the linear relaxation of FC-SSIRP can be shown to be tighter than the linear relaxation of BC-SSIRP. In Figure 5 we illustrate the optimal solution of the linear relaxation of FC-SSIRP for the same example as the one depicted in Figure 4. We can see that the fractional solution satisfies the equilibrium of the flow along each fractional ship path.

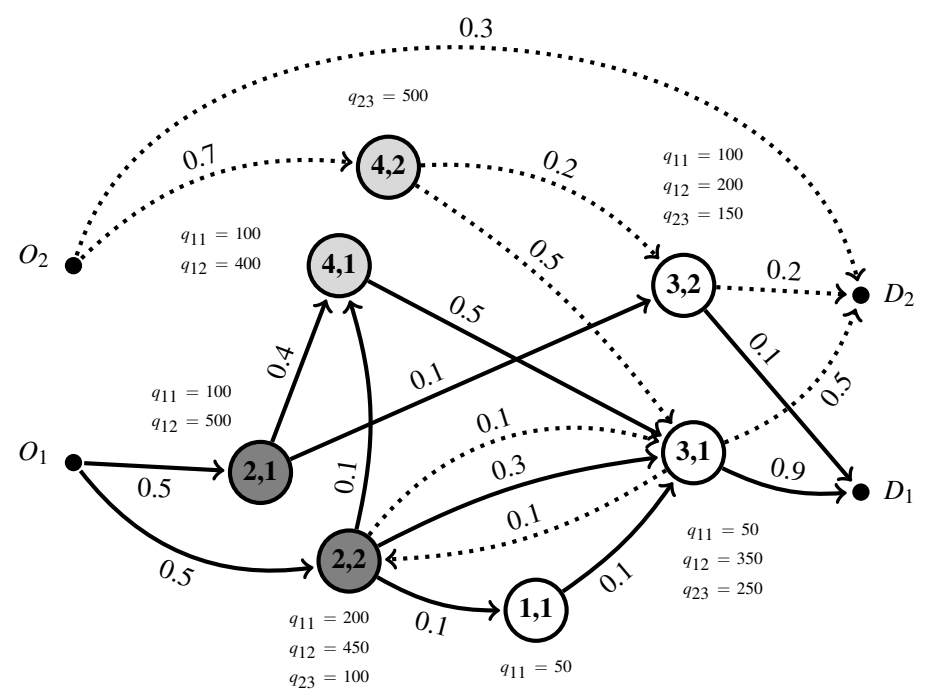

Fig. 5 Optimal solution of the linear relaxation of FC-SSIRP for the example used in Figure 4. In this solution all unloaded products are previously loaded. The quantities $q_{v k}$ represent the quantity of product $k$ loaded (if $k \in\{1,2\}$ and $i=2$, or $k=3$ and $i=4$ ) or unload (in the remaining cases) by ship $v$. 
3.2 Multi-Commodity Reformulations

A multi-commodity reformulation of a flow formulation can be obtained by disaggregating the flow on each arc according to its destination. In general, such types of formulations lead to better bounds.

\subsubsection{Multi-commodity discrete time reformulation}

In this section we define the multi-commodity discrete time formulation with constant consumption rates (MD-SSIRP). By adding new indices to the flow variables indicating the destination of the flow, we construct the non-negative multi-commodity arc-load flow variables $v_{i t j u v k p e}^{D}$, representing the amount of product $k$ that ship $v$ transports from port $i$, after an operation that started in period $t$, to port $j$ for an operation starting in period $u$ to be delivered at port $p$ in period $e$.

These variables are nonnegative

$$
v_{i t j u v k p e}^{D} \geq 0, \quad \forall v \in V, i, j, p \in N, t, u, e \in T, k \in K_{v}: J_{p k}=-1,
$$

and can be related to the arc-load flow variables through the following equations,

$$
f_{i t j u v k}^{D}=\sum_{p \neq i} \sum_{e \geq u} v_{i t j u v k p e}^{D}, \quad \forall v \in V, i, j \in N, t, u \in T, k \in K_{v} .
$$

The tightening of FD-SSIRP can be obtained by replacing constraints (70) with

$$
v_{i t j u v k p e}^{D} \leq \min \left\{C_{v k}, \bar{Q}_{p k}\right\} x_{i t j u v}^{D}, \quad \forall v \in V, i, j, p \in N, t, u, e \in T, k \in K_{v}: J_{p k}=-1 .
$$

The MD-SSIRP can be obtained from the FD-SSIRP by replacing (70) with (77)-(79). Of course the arc-load flow variables $f_{i t j u v k}^{D}$ can be eliminated from the model using (78).

\subsubsection{Multi-commodity continuous time flow reformulation}

Now we define a similar multi-commodity flow formulation for the continuous time model, denoted by MC-SSIRP. We define $v_{i m j n v k p l}^{C}$ as the amount of product $k$ destined to port visit $(p, l)$, which is transported from port visit $(i, m)$ to port visit $(j, n)$ using ship $v$. These variables are nonnegative,

$$
v_{\text {imjnvkpl }}^{C} \geq 0, \quad \forall v \in V,(i, m, j, n) \in S_{v}^{X},(p, l) \in S_{v}^{A}, k \in K_{v}: J_{p k}=-1,
$$

and can be related to the arc-load flow variables by the following equations

$$
f_{\text {imjnvk }}^{C}=\sum_{(p, l) \in S_{v}^{A}: J_{p k}=-1} v_{\text {imjnvkpl }}^{C}, \quad \forall v \in V,(i, m, j, n) \in S_{v}^{X}, k \in K_{v} .
$$

The tightening of the FC-SSIRP can be obtained by replacing constraints (75) by

$$
v_{\text {imjnvkpl }}^{C} \leq \min \left\{C_{v k}, \bar{Q}_{p k}\right\} x_{i m j n v}^{C}, \forall v \in V,(i, m, j, n) \in S_{v}^{X},(p, l) \in S_{v}^{A}, k \in K_{v}: J_{p k}=-1 .
$$

The MC-SSIRP can be obtained from the FC-SSIRP by replacing (75) with (80)-(82). Of course the arc-load flow variables $f_{i m j n v k}^{C}$ can be eliminated from the model using (81). 
4 Tightening the Models

The formulations discussed in Sections 2 and 3 can be strengthened by including valid inequalities and by tightening some constraints. The ideas employed in these improvements are similar for both types of formulations. However, the discrete model embeds time specific information in the network structure that makes the model more amenable for tightening and preprocessing. We discuss only the case with constant consumption rates. The inequalities used in this paper impose either a minimum number of visits to ports or a minimum number of (un)loads. Similar valid inequalities have been used in related papers for constant rate case and for the non constant consumption rates case; see for the last case [1,2,16,20,29]. When consumptions are not constant during time, inequalities based on lot-sizing relaxations have been used, see $[1,2,16]$.

\subsection{Valid Inequalities}

Here we discuss valid inequalities for the models derived in the previous sections. These inequalities allow us to reduce the integrality gap of the proposed models. Hence, although the linear relaxations tend to become more time consuming to solve with the inclusion of these cuts, the reduction of the integrality gap tends to reduce the number of nodes in a branch and bound scheme. The gain in the reduced size of the branch and bound tree compensates the time increase required to obtain the dual bound at each node.

Here we just discuss a type of valid inequalities that impose visits to ports. These visits are forced by the inventory levels combined with the consumption rates. First we consider the discrete time models BD-SSIRP, FD-SSIRP, and MD-SSIRP.

For each unloading (consumption) port $i \in N$ and product $k, J_{i k}=-1$, let

$$
N D_{i k}=\max \left\{\bar{T} \times R_{i k}-S_{i k}^{O}+\underline{S}_{i k}, \quad 0\right\}
$$

denote the net consumption or demand over the time horizon. If $0<N D_{i k}<\underline{Q}_{i k}$, then the net demand can be increased to the minimum load quantity: $N D_{i k}=\underline{Q}_{i k}$. The minimum number of visits at port $i$ for unloading product $k$ is given by

$$
\underline{\lambda}_{i k}=\left\lceil\frac{N D_{i k}}{\bar{Q}_{i k}}\right\rceil .
$$

Hence, the following inequalities are valid

$$
\begin{aligned}
& \sum_{v \in V} \sum_{j \in N} \sum_{u \in T} \sum_{t \in T} x_{j u i t v}^{D} \geq \underline{\lambda}_{i k}, \quad \forall i \in N, k \in K: J_{i k}=-1, \\
& \sum_{v \in V} \sum_{t \in T} o_{i t v k}^{D} \geq \underline{\lambda}_{i k}, \quad \forall i \in N, k \in K: J_{i k}=-1 .
\end{aligned}
$$

These inequalities can be generalized for each period $t \in T$, as follows. We split the time horizon into two periods, one from 0 to the end of period $t$ and the other from $t$ to the end of the time horizon. Let

$$
N D_{i t k}^{0}=t \times R_{i k}-S_{i k}^{O}+\underline{S}_{i k},
$$

be the net consumption until the end of period $t$ and let

$$
\underline{N D_{i t k}^{\bar{T}}}=(\bar{T}-t+1) \times R_{i k}-\bar{S}_{i k}+\underline{S}_{i k},
$$


be an underestimation of the net consumption from the end of period $t$ until the end of the time horizon. Define

$$
\varepsilon_{i t k}^{0}=\left\lceil\frac{N D_{i t k}^{0}}{\bar{Q}_{i k}}\right\rceil,
$$

and

$$
\varepsilon_{i t k}^{\bar{T}}=\left\lceil\frac{N D_{i t k}^{T}}{\bar{Q}_{i k}}\right\rceil,
$$

as a lower bound on the number of visits to port $i$. Then the following inequalities are valid

$$
\begin{aligned}
& \sum_{u \in T \mid u \leq t} \sum_{j \in N} \sum_{e \in T} \sum_{v \in V} x_{\text {jeiuv }}^{D} \geq \varepsilon_{i t k}^{0}, \quad \forall i \in N, t \in T, k \in K: J_{i k}=-1, \\
& \sum_{u \in T \mid u \leq t} \sum_{v \in V} o_{i u v k}^{D} \geq \varepsilon_{i t k}^{0}, \quad \forall i \in N, t \in T, k \in K: J_{i k}=-1, \\
& \sum_{u \in T \mid u>t} \sum_{j \in N} \sum_{e \in T} \sum_{v \in V} x_{j e i u v}^{D} \geq \varepsilon_{i t k}^{\bar{T}}, \quad \forall i \in N, t \in T, k \in K: J_{i k}=-1, \\
& \sum_{u \in T \mid u>t} \sum_{v \in V} o_{i u v k}^{D} \geq \varepsilon_{i t k}^{T}, \quad \forall i \in N, t \in T, k \in K: J_{i k}=-1 .
\end{aligned}
$$

In order to ensure that if $\operatorname{ship} v$ unloads product $k$ at port $i$ in period $t$, then there must exist a route of $\operatorname{ship} v$ passing through port $i$ at period $t$, the following inequalities can be added:

$$
o_{i t v k}^{D} \leq \sum_{j \in N} \sum_{u \in T} x_{j u i t v}^{D}, \quad \forall v \in V, i \in N, t \in T, k \in K_{v}: J_{i k}=-1
$$

Inequalities (89) coupled with constraints (84) imply (83). This is no longer true if we consider in (83) the aggregated demand (consumption) of a subset of consumption ports instead of the demand of port $i$ only.

In the underlying real planning problem, the inventory bounds are usually not tight for the loading ports. Hence, the minimum number of departures can be estimated using the total demand supplied by those ports. In the real problem, each product has a single origin, so the demand of that product must be satisfied either from that port or from the quantity in the ship tanks at the beginning of the time horizon.

For each product $k \in K$ and loading port $i \in N\left(J_{i k}=1\right)$, let

$$
N D_{i k}=\sum_{j \in N \mid J_{j k}=-1}\left(\bar{T} \times R_{j k}-S_{i k}^{O}+\underline{S}_{i k}\right)-\sum_{v \in V} Q_{v k},
$$

denote the demand (consumption) in excess of what is available on board the ships in the beginning of the planning horizon. The minimum number of loadings of product $k$ at port $i$ is given by

$$
\underline{\lambda}_{i k}=\left\lceil\frac{N D_{i k}}{\max \left\{C_{v k}: v \in V\right\}}\right\rceil .
$$

Hence, the following inequalities are valid

$$
\begin{aligned}
& \sum_{v \in V} \sum_{j \in N} \sum_{u \in T} \sum_{t \in T} x_{j u i t v}^{D} \geq \underline{\lambda}_{i k}, \quad \forall i \in N, k \in K: J_{i k}=1, \\
& \sum_{v \in V} \sum_{t \in T} o_{i t v k}^{D} \geq \underline{\lambda}_{i k}, \quad \forall i \in N, k \in K: J_{i k}=1 .
\end{aligned}
$$


As done for the consumption ports, we can derive inequalities for each period $u$ for the loading ports as well; see (85) - (89). We omit these inequalities here.

Observe that a lower bound on the total number of visits to port $i \in N$ can be given by

$$
\underline{\mu}_{i}=\max \left\{\underline{\lambda}_{i k}: k \in K\right\} .
$$

Hence, the following inequalities are valid:

$$
\sum_{t \in T} y_{i t}^{D} \geq \underline{\mu}_{i}, \quad \forall i \in N
$$

Now we consider the continuous models BC-SSIRP, FC-SSIRP and MC-SSIRP. Here we can only impose a minimum number of visits during the planning horizon since the order of the visits does not provide information about the time for start of service at the visit. Inequalities (83)-(84) for the consumption ports and (90)-(91) for the loading/production ports can be written for the continuous case as follows:

$$
\begin{aligned}
& \sum_{v \in V} \sum_{(j, n) \in S_{v}^{A}} \sum_{m \in\left\{1, \ldots, \bar{\mu}_{i}\right\}} x_{\text {jnimv }}^{C} \geq \underline{\lambda}_{i k}, \quad \forall i \in N, k \in K, \\
& \sum_{v \in V} \sum_{m \in\left\{1, \ldots, \bar{\mu}_{i}\right\}} o_{i m v k}^{C} \geq \underline{\lambda}_{i k}, \quad \forall i \in N, k \in K .
\end{aligned}
$$

In the continuous time case, the lower bound on the number of visits can be imposed by the inequality

$$
y_{\underline{i}_{i}}^{C}=1, \quad i \in N .
$$

\subsection{Tightening constraints}

Now we consider another approach to strengthen the models by tightening the linking constraints. The linking constraints relate the continuous variables to the binary variables. Improving these constraints can lead to reductions in the integrality gap and in running times. We focus on formulations for the constant consumption rate case only.

First we consider the tightening of constraints (24) for the discrete model and (58) for the continuous model, linking time variables with routing variables. The main idea is to aggregate the routing variables for $v$ since the time variables do not depend on the particular ship $v$. Consider the time constraints (24) for the discrete model. These inequalities can be replaced by the following ones

$$
t_{i t}^{E D}+\sum_{v \in V} T_{i j v} x_{i t j u v}^{D}-t_{j u}^{D} \leq \bar{T}\left(1-\sum_{v \in V} x_{i t j u v}^{D}\right), \quad \forall i, j \in N, t, u \in T .
$$

When time windows are established to time events

$$
\begin{aligned}
& A_{i t} \leq t_{i t}^{D} \leq B_{i t}, \quad \forall i \in N, t \in T, \\
& A_{i t}^{E} \leq t_{i t}^{E D} \leq B_{i t}^{E}, \quad \forall i \in N, t \in T,
\end{aligned}
$$

then, constraints (24) can be replaced by inequalities

$$
t_{i t}^{D E}-t_{j u}^{D}+\left(B_{i t}^{E}+T_{i j v}-A_{j u}\right) x_{i t j u v} \leq B_{i t}^{E}-A_{j u}, \quad \forall v \in V, i, j \in N, t, u \in T .
$$


These inequalities can be further strengthened as follows (see Proposition 1 in [4]):

$$
t_{i t}^{D E}-t_{j u}^{D}+\sum_{v \in V} \max \left\{0, B_{i t}^{E}+T_{i j v}-A_{j u}\right\} x_{i t j u v} \leq B_{i t}^{E}-A_{j u}, \forall i, j \in N, t, u \in T .
$$

Constraints (25) establish time windows for $t_{i t}^{D}$. For $t_{i t}^{E D}$ we assume $A_{i t}^{E}=t-1$ and $B_{i t}^{E}=t+1$ since an operation takes at most one time period (day).

For the continuous models, constraints (58) can be strengthened in a similar way. We omit the details here. The major difference is related to the computation of time windows $\left[A_{i m}, B_{i m}\right]$ for $t_{i m}^{C}$, and $\left[A_{i m}^{E}, B_{i m}^{E}\right]$ for $t_{i m}^{E C}$. First we set $A_{i m}=A_{i m}^{E}=0$ and $B_{i m}=B_{i m}^{E}=T$. By reducing the widths of these time windows we strengthen the resulting inequality. However, since we are dealing with multiple ships, multiple products, and all supply ports also act as demand ports of other products, it is hard to derive tight time windows. Additionally, some preliminary results showed that small improvements in the widths of time windows do not lead to any practical gain.

Next we consider another tightening which use information of the demands to tighten the linking coefficients. For instance, consider inequalities (13) in model BD-SSIRP. The unload quantity at period $t$ can be additionally limited by the remaining consumption at that port. That is,

$$
q_{i t v k}^{D} \leq \min \left\{C_{v k}, \bar{Q}_{i k}, A\right\} o_{i t v k}^{D}, \quad \forall v \in V, i \in N, t \in T, k \in K_{v}: J_{i k}=-1,
$$

where $A=\max \left\{R_{i}^{k}(\bar{T}-t+1), \underline{Q}_{i k}\right\}$.

For the BC-SSIRP model, the corresponding variables, $q_{i t v k}^{C}$, do not provide information of time of the visit. So we can only limit the demand/consumption for the total time horizon.

Similar reasoning can be applied to inequalities (11), (12), (18)-(21). For brevity we give the tightening for the flow and multi-commodity formulations in more detail only.

Consider the arc-load flow models FD-SSIRP and FC-SSIRP. In FD-SSIRP, inequalities (70) can be replaced by

$$
f_{i t j u v k}^{D} \leq \min \left\{C_{v k}, B 1\right\} x_{i t j u v}^{D}, \quad \forall v \in V, i, j \in N, t, u \in T, k \in K_{v},
$$

where $B 1=\sum_{j \in N \mid J_{j k}=-1} \max \left\{R_{j k}(\bar{T}-u+1), \underline{Q}_{j k}\right\}$. In FC-SSIRP, inequalities (75) can be replaced by

$$
f_{\text {imjnvk }}^{C} \leq \min \left\{C_{v k}, B 2\right\} x_{\text {imjnv }}^{C}, \quad \forall v \in V,(i, m, j, n) \in S_{v}^{X}, k \in K_{v},
$$

where $B 2=\max \left\{\sum_{j \in N \mid J_{j k}=-1} R_{j k} \bar{T}, \underline{Q}_{j k}\right\}$.

Now consider the multi-commodity flow models MD-SSIRP and MC-SSIRP. In MDSSIRP, inequalities (79) can be replaced by

$$
v_{i t j u v k p e}^{D} \leq \min \left\{C_{v k}, \bar{Q}_{p k}, C 1\right\} x_{i t j u v}^{D}, \forall v \in V, i, j, p \in N, t, u, e \in T, k \in K_{v}: J_{p k}=-1,
$$

where $C 1=\max \left\{R_{p k}(\bar{T}-u+1), \underline{Q}_{p k}\right\}$. In MC-SSIRP, inequalities (82) can be replaced by

$$
\begin{aligned}
v_{\text {imjnvkpl }}^{C} \leq \min \left\{C_{v k}, \bar{Q}_{p k}, C 2\right\} x_{\text {imjnv }}^{C}, \quad & \forall v \in V,(i, m, j, n) \in S_{v}^{X},(p, l) \in S_{v}^{A}, \\
& k \in K_{v}: J_{p k}=-1,
\end{aligned}
$$


where $C 2=\max \left\{R_{p k} \bar{T}, Q_{p k}\right\}$.

We can see that $B 1$ and $C 1$ depend on the time period, while $B 2$ and $C 2$ do not. This is one of the advantages of the discrete models.

\section{Computational Experiments}

In this section we conduct computational experiments to test and compare the discrete time and the continuous time models. All computations were performed using the optimization software Xpress Optimizer Version 20.00.05 with Xpress Mosel Version 3.0.0, on a computer with an Intel Core 2 Duo processor, with CPU $2.2 \mathrm{GHz}$, and with 4GB of RAM.

We use two sets of instances for the SSIRP with constant consumption rates. The first set consists of 12 real instances from a company in Cape Verde including 2 ships, 4 products and 7 ports. The other set consists of 12 instances from an artificial scenario where the consumption rates of the real instances are doubled as well as the number of ships.

First we describe some characteristics of the instances. The typical planning horizon is two weeks. Here we consider instances with $\bar{T}=10$ and $\bar{T}=15$. The demand for each product during the planning horizon is, in average, 2.5 times the largest ship tank capacity. The tank capacity at the main ports can cover the demand at that port for a week (without regard the safety stocks). For the small islands typically one or two visits are required. The total number of visits for the tested instances ranged between 12 and 15. The ships have in average 6 tanks.

Computational experiments are conducted to compare the models according to their size, running times and integrality gap without any additional tightening. Based on the information obtained, we select some of the models for further testing. The selected models are used in a branch-and-cut scheme to solve the two sets of instances.

We also tested the influence of the minimum unload values $Q_{i k}$ on solution quality and tractability.

\subsection{Comparison of the Size of the Models}

Now we compare the size of the models without any tightening or addition of cuts. Table 1 provides the information of the average number of variables and average number of constraints of the three discrete time and continuous time formulations for a time horizon of 10 and 15 periods (days). Additionally, column "Solved" gives the number of instances solved to optimality using the default options of Xpress optimizer within a time limit of 3 hours.

For the discrete time models we ignore all variables $x_{i t j u v}^{D}$ with $u>t+3$, and for the continuous time model we established the upper bound of the number of visits to port $i$, $\bar{\mu}_{i}=\underline{\mu}_{i}+3$.

$\overline{\mathrm{We}}$ can see that each continuous time model is smaller than the corresponding discrete time model. Table 1 also shows that multi-commodity models are too large and most of the larger instances cannot be solved within the time limit of 3 hours.

Next we study the impact of eliminating some arc-load variables in both types of models. For the discrete time models we eliminate all variables $x_{i t j u v}^{D}$ with $u>t+\alpha$, and for the continuous time models we established the upper bound of the number of visits to port $i$, as $\bar{\mu}_{i}=\mu_{i}+\alpha$. If $\alpha$ is small we reduce substantially the set of feasible solutions and it is 
Table 1 Average size of the tested models.

\begin{tabular}{|c|c|c|c|c|c|c|c|c|}
\hline \multicolumn{2}{|r|}{ Model } & $\bar{T}$ & $\mid \mathrm{VI}$ & Binary Var. & Cont. Var. & Total Var. & Constraints & Solved \\
\hline \multirow{12}{*}{ 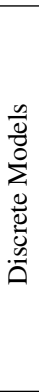 } & BD-SSIRP & 10 & 2 & 3636 & 1185 & 4821 & 22854 & 10 \\
\hline & FD-SSIRP & 10 & 2 & 3636 & 7975 & 11611 & 13334 & 9 \\
\hline & MD-SSIRP & 10 & 2 & 3636 & 155955 & 159591 & 111668 & 7 \\
\hline & BD-SSIRP & 10 & 4 & 7392 & 2533 & 9925 & 74772 & 4 \\
\hline & FD-SSIRP & 10 & 4 & 7392 & 14209 & 21601 & 27928 & 10 \\
\hline & MD-SSIRP & 10 & 4 & 7392 & 311975 & 319367 & 227748 & 2 \\
\hline & BD-SSIRP & 15 & 2 & 5706 & 1775 & 7481 & 36044 & 5 \\
\hline & FD-SSIRP & 15 & 2 & 5706 & 12590 & 18296 & 20924 & 9 \\
\hline & MD-SSIRP & 15 & 2 & 5706 & 370570 & 376276 & 254543 & 2 \\
\hline & BD-SSIRP & 15 & 4 & 11592 & 3783 & 15375 & 159917 & 4 \\
\hline & FD-SSIRP & 15 & 4 & 11592 & 22004 & 33596 & 49933 & 7 \\
\hline & MD-SSIRP & 15 & 4 & 11592 & 741240 & 752832 & 525598 & 2 \\
\hline \multirow{12}{*}{$\begin{array}{l}\frac{n}{0} \\
\frac{0}{0} \\
\sum_{0}^{0} \\
0 \\
0 \\
.0 \\
0 \\
0 \\
0\end{array}$} & BC-SSIRP & 10 & 2 & 2356 & 606 & 2962 & 15288 & 12 \\
\hline & FC-SSIRP & 10 & 2 & 2356 & 5376 & 7732 & 8668 & 12 \\
\hline & MC-SSIRP & 10 & 2 & 2356 & 36896 & 39252 & 46085 & 12 \\
\hline & BC-SSIRP & 10 & 4 & 3278 & 960 & 4238 & 22411 & 4 \\
\hline & FC-SSIRP & 10 & 4 & 3278 & 8000 & 11278 & 12511 & 12 \\
\hline & MC-SSIRP & 10 & 4 & 3278 & 41908 & 45186 & 55006 & 3 \\
\hline & BC-SSIRP & 15 & 2 & 2484 & 623 & 3107 & 16153 & 6 \\
\hline & FC-SSIRP & 15 & 2 & 2484 & 5678 & 8162 & 9133 & 11 \\
\hline & MC-SSIRP & 15 & 2 & 2484 & 39074 & 41558 & 48726 & 2 \\
\hline & BC-SSIRP & 15 & 4 & 3926 & 1065 & 4991 & 27214 & 4 \\
\hline & FC-SSIRP & 15 & 4 & 3926 & 9656 & 13582 & 15004 & 8 \\
\hline & MC-SSIRP & 15 & 4 & 3926 & 51004 & 54930 & 66596 & 2 \\
\hline
\end{tabular}

possible that the instance becomes infeasible. On the other hand if $\alpha$ is large the size of the model increases and the running times tend to be very high. In order to illustrate the effects of $\alpha$ on the optimal solution, we tested the set of 12 real instances with 10 and 15 periods. Each instance was solved for $\alpha$ from 1 to 3. The results are given in Table 2. The table gives the number of instances that resulted in the true optimal value using models FD-SSIRP and FC-SSIRP.

Table 2 Number of instances where the true optimal solution was obtained. All instances were solved to optimality. We considered $|V|=2$.

\begin{tabular}{|c|c|c|c|c|}
\hline & \multicolumn{2}{|c|}{ FD-SSIRP } & \multicolumn{2}{c|}{ FC-SSIRP } \\
\hline$\alpha$ & $\bar{T}=10$ & $\bar{T}=15$ & $\bar{T}=10$ & $\bar{T}=15$ \\
\hline 1 & 5 & 0 & 2 & 0 \\
2 & 11 & 2 & 12 & 11 \\
3 & 12 & 12 & 12 & 12 \\
\hline
\end{tabular}

For $\alpha=1$ the optimal value is worse compared to the true optimal value in most instances. This situation is opposite for $\alpha=2$. For $\alpha=3$ we obtain the true optimal value for all the tested instances. A more detailed test (not reported here) revealed that in order to keep the quality of the optimal solution while minimizing the number of variables, for continuous time models, different values of $\alpha$ can be chosen for different ports. Small values of $\alpha$ can be assumed for low activity ports while larger values should be assumed for high activity ones. Additionally, Table 2 shows that when the length of the planning horizon is increased the value of $\alpha$ should also increase to obtain the optimal solution. 
Figure 6 shows the average running times of the arc-load flow models FD-SSIRP and FC-SSIRP (which proved to be the fastest models among all the tested models) when $\alpha$ varies from 1 to 5. It is clear that the running time increases rapidly with the increase of $\alpha$, and the running times of the discrete time model increase faster than the running time of the continuous time model.
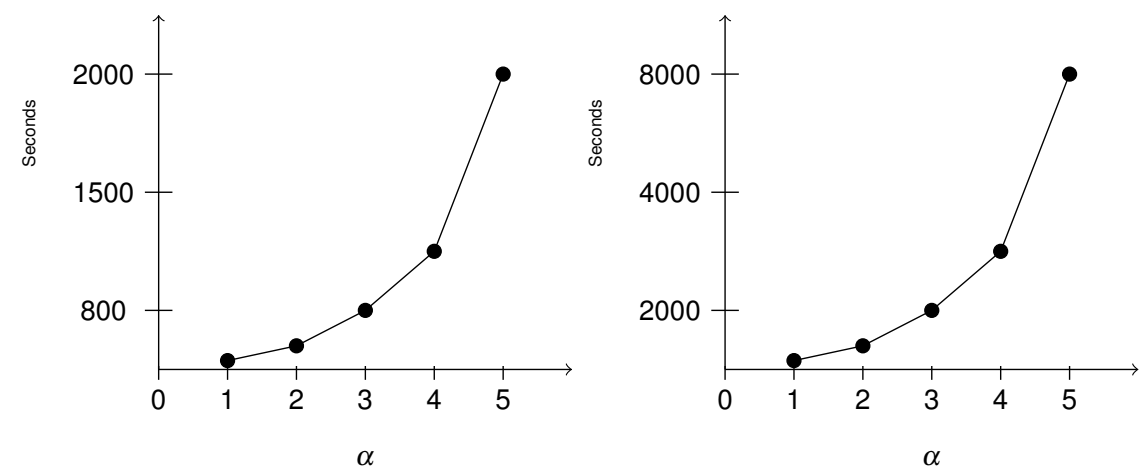

Fig. 6 Average solution times using the arc-load flow formulations (FC-SSIRP on left and FD-SSIRP on right) on 12 real instances with $\bar{T}=10$, and $|V|=2$, when increasing $\alpha$.

\subsection{Comparison of the Integrality Gaps}

Next we present some computational results in order to compare the integrality gap of the various formulations. The results of the set of real instances are reported in Table 3. For each formulation we present the average integrality gap at the root node, gap = $\frac{\text { Optimal value }- \text { Lower Bound }}{\text { Optimal value }} \times 100$ for several possible settings. Column $N$ means the original formulation without tightening of constraints and without inclusion of cuts; Column TT means with tightening only; Column $C$ means with inclusion of cuts; and Column $T T+C$ means with tightening and inclusion of cuts. When cuts are added we indicate the average number of cuts added (Column Ncuts). Notice that the lower bounds obtained without valid inequalities and tightening are very poor, especially for the arc-load formulations, BD-SSIRP and BC-SSIRP. We can observe that strengthening the models with the addition of inequalities (83), (84), (90), (91) and with the tightening of constraints reduces the integrality gaps considerably. Finally, we observe that the arc-load and the arc-load flow formulations for $N$ and $C$ cases provide essentially the same bounds for both approaches (discrete time and continuous time). With the inclusion of valid inequalities and tightening of constraints the discrete time models provide slightly better gaps than the corresponding continuous time models. This is explained by the fact that, in discrete time models we can provide tighter constraints as explained in Section 4.2.

We conduct similar computational experiments for the set of artificial instances with 4 ships, 4 products and 7 ports, where the consumption rate is doubled. Here we report results obtained with the models FD-SSIRP-C and FC-SSIRP only, since the running time was limited to three hours and the multi-commodity formulations are very time consuming. 
Table 3 Average integrality gaps with and without tightening of constraints and inclusion of valid inequalities. We considered $|V|=2$.

\begin{tabular}{|c|c|c|c|c|c|c|c|c|c|c|c|c|}
\hline & \multicolumn{3}{|c|}{$\mathrm{N}$} & \multicolumn{2}{|c|}{ TT } & \multicolumn{3}{c|}{$\mathrm{C}$} & \multicolumn{3}{c|}{ TT+C } \\
\hline Model & $\bar{T}=10$ & $\bar{T}=15$ & $\bar{T}=10$ & $\bar{T}=15$ & $\bar{T}=10$ & Ncuts & $\bar{T}=15$ & Ncuts & $\bar{T}=10$ & Ncuts & $\bar{T}=15$ & Ncuts \\
\hline \hline BD-SSIRP & 57.6 & 45.3 & 55.6 & 43.2 & 9.2 & 51 & 26.1 & 151.1 & 7.9 & 42.3 & 25.8 & 108.3 \\
FD-SSIRP & 48.9 & 31.4 & 47.5 & 31.1 & 6.5 & 60.9 & 16.1 & 151.1 & 3.1 & 39.1 & 13.3 & 94.5 \\
MD-SSIRP & 43.3 & 26 & 41.3 & 22.8 & 6.5 & 68.3 & 16.1 & 151.2 & 3.1 & 65.1 & 13.1 & 150 \\
\hline \hline BC-SSIRP & 57.6 & 45.3 & 57.6 & 45.3 & 9.2 & 12.5 & 26.8 & 15.3 & 7.9 & 12.5 & 25.8 & 14.9 \\
FC-SSSRP & 48.9 & 31.4 & 48.9 & 31.4 & 6.5 & 12.1 & 16.7 & 13.8 & 3.1 & 10.3 & 15.1 & 13.5 \\
MC-SSIRP & 43.3 & 26 & 41.3 & 23.6 & 6.5 & 15.2 & 17.4 & 13.7 & 3.1 & 10.3 & 14.8 & 13.5 \\
\hline
\end{tabular}

The results for these two models, including tightening constraints and cuts, are presented in Table 4. We give the average initial integrality gap (Gap-I), that is, the average of the integrality gaps at the root node, the average gap provided by Xpress after the three hours limit (Gap-E), and the average running time (Time). We can see that the average initial gap is smaller using FD-SSIRP but the running times are smaller using the continuous model FC-SSIRP.

Table 4 Average computational results for FD-SSIRP and FC-SSIRP with $|V|=4$.

\begin{tabular}{|c|c|c|c|c|c|c|}
\hline & \multicolumn{3}{|c|}{ FD-SSIRP-C } & \multicolumn{3}{c|}{ FC-SSIRP } \\
\hline & Gap-I & Gap-E & Time (sec.) & Gap-I & Gap-E & Time (sec.) \\
\hline \hline $\bar{T}=10$ & 12.9 & 0 & 907 & 13.9 & 0 & 476 \\
\hline \hline $\bar{T}=15$ & 15.4 & 5.3 & 6172 & 17.8 & 2.4 & 5602 \\
\hline
\end{tabular}

\subsection{Impact of Minimum Delivery Quantities}

Restrictions on the minimum delivery quantities of each product at each port are considered for the SSIRP with constant consumption rates. In fact, delivering small quantities may result in too many port visits. In reality one wants to avoid too many visits to a port due to issues like unpredictable weather conditions and port occupancy. Based on historical data of real instances we conclude that the minimum allowed delivery quantities, $\underline{Q}_{i k}$, are around $40 \%$ of the maximum allowed unloading quantities, $\bar{Q}_{i k}$. In order to analyze the real impact of $\underline{Q}_{i k}$, in the objective function value, integrality gap, running time, and the number of branch and bound nodes, we solve the 12 real instances for different values of $\underline{Q}_{i k}$, ranging from $0 \%$ to $90 \%$ of $\bar{Q}_{i k}$, using the FC-SSIRP model. The results are presented in Figures 7 and 8 and show that when $Q_{i k}$, varies from $0 \%$ to $60 \%$ the cost increases slowly, but when it is greater than $60 \%$ the cost increases significantly. We also observe that time, integrality gap and number of nodes, have small oscillation until $60 \%$, increase significantly between $60 \%$ and $80 \%$, and decrease after $80 \%$.

\subsection{Comparison of the Running Times and Number of Branch and Bound Nodes}

From Section 5.1 we see that the multi-commodity formulations are much larger in number of variables and constraints than the arc-load and arc-load flow formulations. However, Section 5.2 shows that the reduction in the integrality gap by using the multi-commodity 

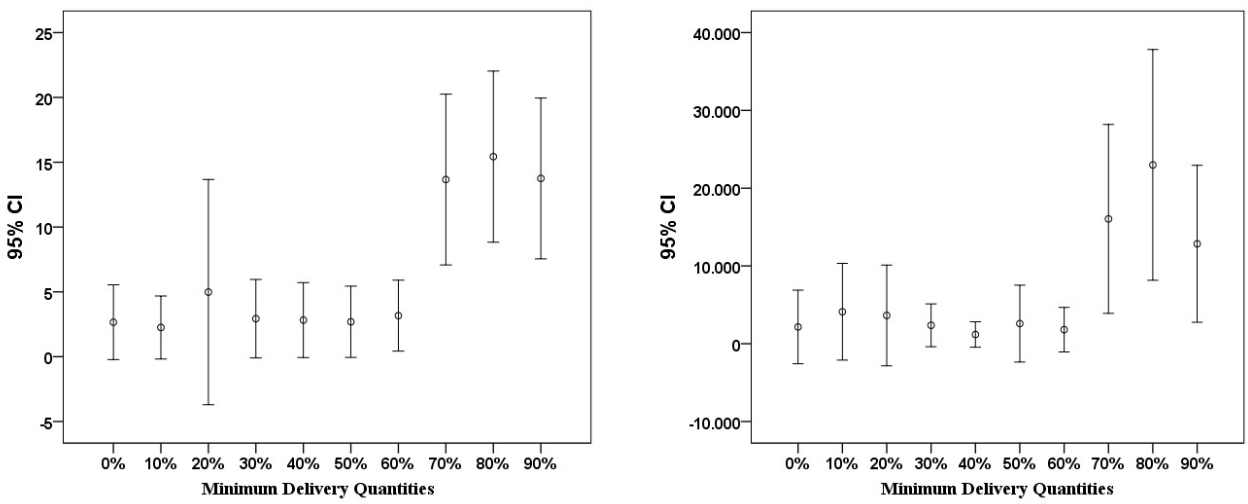

Fig. 7 Impact of minimum delivery quantities on the integrality gap (left) and number of branch and bound nodes (right).
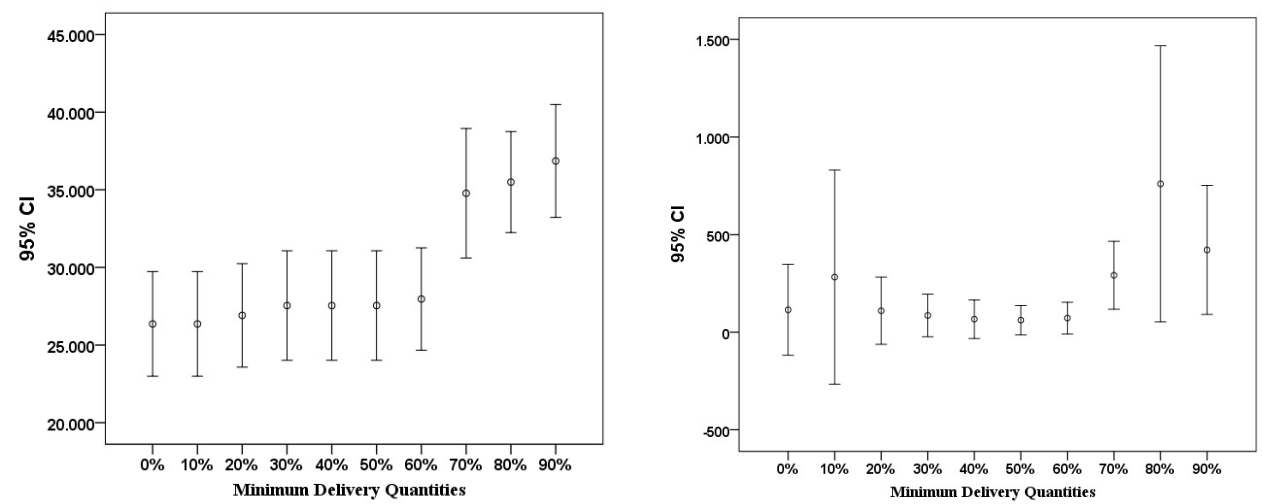

Fig. 8 Impact of minimum delivery quantities on the solution cost (left) and on the running time (right).

formulations is very small. These two observations lead to the conclusion that the multicommodity formulations can hardly be competitive compared to the other two formulations. Preliminary results, not reported here, confirm this conclusion. Therefore, in this section we report results for the BD-SSIRP (BC-SSIRP) and FD-SSIRP (FC-SSIRP) models.

A comparison of the running times and number of branch and bound nodes using the BD-SSIRP (BC-SSIRP) and FD-SSIRP (FC-SSIRP) models, for each approach, is shown in Table 5. The notation is the same as the one for Table 3. For $\bar{T}=15$, only results with tightening and inclusion of cuts are presented because most of the instances were not solved within 3 hours for the remaining cases. The tests were performed for the 12 real instances. We observe that tightening constraints and including cuts is essential when solving the instances. The best results where obtained with the improved (with tightened constraints and cuts) FD-SSIRP and FC-SSIRP models. In fact, only this combination allowed us to solve all the tested instances to optimality. We can see that in several cases the number of branch and bound nodes was smaller using the discrete models. This can be justified by the fact that the discrete time model has, on average, slightly better integrality gaps. However, the 
Table 5 Average running times and number of branch and bound nodes. The running time was set to 3 hours when the instance was not solved within the 3 hours limit.

\begin{tabular}{|c|c|c|c|c|c|c|c|c|c|c|}
\hline & \multicolumn{9}{|c|}{$\bar{T}=10$} & \multicolumn{2}{c|}{$\bar{T}=15$} \\
\hline & \multicolumn{2}{|c|}{$\mathrm{N}$} & \multicolumn{2}{|c|}{ TT } & \multicolumn{2}{c|}{ C } & \multicolumn{2}{c|}{ TT+C } & \multicolumn{3}{c|}{ TT+C } \\
\hline & Time & Nodes & Time & Nodes & Time & Nodes & Time & Nodes & Time & Nodes \\
\hline BD-SSIRP & 743 & 38391 & 1090 & 26993 & 590 & 26493 & 412 & 26942 & 6305 & 37236 \\
FD-SSIRP & 1614 & 32249 & 1347 & 16035 & 619 & 8537 & 86 & 916 & 3773 & 37213 \\
\hline \hline BC-SSIRP & 487 & 85695 & 360 & 85395 & 112 & 29453 & 84 & 14839 & 3091 & 36976 \\
FC-SSIRP & 245 & 26823 & 78 & 4320 & 84 & 8120 & 39 & 3544 & 2740 & 36926 \\
\hline
\end{tabular}

continuous time model was clearly faster than the discrete one. If we recall that the size of the continuous model is smaller than the size of the discrete one, and the difference on the average integrality gaps is small, we may conclude that this is the expected behavior of the two models, that is, the continuous model should outperform the discrete model, and this difference tends to be larger when $\bar{T}$ increases.

\section{Conclusions}

We present a real short sea inventory routing problem for fuel oil distribution. We provide two types of formulations. A discrete time model for both time varying and constant consumption, and a continuous time model for constant consumption rates. We discuss different extended formulations for both types of formulations, and valid inequalities that allow us to derive tighter formulations.

All the models proposed were compared according to their size, integrality gap and running time using a commercial software. From this comparison we conclude that: i) the extended formulations based on arc-load flow variables with valid inequalities provide the best compromise between integrality gaps and size of model; ii) the discrete time models tend to provide better bounds. However, the running times using the discrete time models are in general worse than the running times using the continuous time model.

From i) and ii) we conclude that, for the constant consumption rate case, the continuous time arc-load flow model with valid inequalities is the best option among all the tested ones to solve small real sized instances. With this formulation we solved instances with up to 15 days to optimality.

\section{Acknowledgements}

This research was carried out with financial support from the portuguese Founda- tion for Science and Technology (FCT) through project UID/MAT/04106/2013 (first and third authors), and from the Research Council of Norway through the DOMinant II project (second author). This support is gratefully acknowledged. 
Appendix A: glossary of problem and model acronyms

Problem acronyms:

SSIRP: $\quad$ Short Sea Inventory Routing Problem with constant consumption rates.

SSIRP-V: Short Sea Inventory Routing Problem with Varying consumption rates.

Model acronyms:

BD-SSIRP-V: Basic arc-load Discrete time model for the SSIRP-V.

BD-SSIRP: $\quad$ Basic arc-load Discrete time model for the SSIRP.

BC-SSIRP: Basic arc-load Continuous time model for the SSIRP.

FD-SSIRP: Arc-load Flow Discrete time model for the SSIRP.

FC-SSIRP: Arc-load Flow Continuous time model for the SSIRP.

MD-SSIRP: Multi-commodity arc-load Discrete time model for the SSIRP.

MC-SSIRP: Multi-commodity arc-load Continuous time model for the SSIRP.

\section{References}

1. Agra, A., Andersson, H., Christiansen, M., Wolsey, L., A maritime inventory routing problem: Discrete time formulations and valid inequalities. Networks, 62, 297-314, (2013).

2. Agra, A., Christiansen, M., Delgado, A., Mixed integer formulations for a short sea fuel oil distribution problem. Transportation Science, 47, 108-124, (2013).

3. Agra, A., Christiansen, M., Delgado, A., Simonetti, L., Hybrid heuristics for a maritime short sea inventory routing problem. European Journal of Operations Research, 236, 924-935, (2014).

4. Agra A., Christiansen M., Hvattum L.M., Figueiredo R., Poss M., Requejo C., The robust vehicle routing problem with time windows. Computers \& Operations Research, 40, 856-866, (2013).

5. Al-Khayyal, F., Hwang, S.-J., Inventory constrained maritime routing and scheduling for multicommodity liquid bulk, Part I: Applications and model. European Journal of Operational Research, 176 (1), 106-130, (2007).

6. Andersson, H., Hoff, A., Christiansen, M., Hasle, G., Løkketangen, A., Industrial aspects and literature survey: Combined inventory management and routing. Computers \& Operations Research, 37 (9), 15151536, (2010).

7. Archetti, C., Bertazzi, L., Hertz, A., Speranza, M.G., A hybrid heuristic for an inventory routing problem. INFORMS Journal on Computing, 34 (1), 101-116, (2012).

8. Christiansen, M., Inventory and Time Constrained Ship Routing: A Mathematical Programming Approach. PhD Thesis, Norwegian University of Science and Technology, Norway, (1996).

9. Christiansen, M., Decomposition of a combined inventory and time constrained ship routing problem. Transportation Science, 33 (1), 3-16, (1999).

10. Christiansen, M., Fagerholt, K., Maritime Inventory Routing Problems. In: Floudas, C., Pardalos, P. (Eds.), Encyclopedia of optimization, second edition. Springer, pp. 1947-1955, (2009).

11. Christiansen, M., Fagerholt, K., Flatberg, T., Haugen, Ø., Kloster, O., Lund, E., Maritime inventory routing with multiple products: A case study from the cement industry. European Journal of Operational Research, 208 (1), 86-94, (2011).

12. Christiansen, M., Fagerholt, K., Nygreen, B., Ronen, D., Maritime Transportation. In: C. Barnhart and G. Laporte (Eds.), Handbooks in Operations Research and Management Science 14, Transportation. North-Holland, Amsterdam, pp. 189-284, (2007).

13. Christiansen, M., Fagerholt, K., Nygreen, B., Ronen, D., Ship routing and scheduling in the new millennium European Journal of Operations Research, 228 (3), 467-483, (2013).

14. Christiansen, M., Fagerholt, K., Ronen, D., Ship routing and scheduling: Status and perspectives. Transportation Science, 38 (1), 1-18, (2004).

15. Desrosiers, J., Dumas, Y., Solomon, M. M., Soumis, F., Time constrained routing and scheduling. In: M. O. Ball, T. L. Magnanti, C. L. M., Nemhauser, G. L. (Eds.), Handbooks in Operations Research and Management Science 8, Network Routing. North-Holland, Amsterdam, pp. 35-139, (1995). 
16. Engineer, F.G., Furman, K.C., Nemhauser, G.L., Savelsbergh, M.W.P., Song, J.-H., A branch-price-andcut algorithm for single-product maritime inventory routing. Operations Research, 60 (1), 106-122, (2012).

17. Flatberg, T., Haavardtun, H., Kloster, O., Løkketangen, A., Combining exact and heuristic methods for solving a vessel routing problem with inventory constraints and time windows. Ricerca Operativa, 29 (91), 55-68, (2000).

18. Furman, K.C., Song, J.-H., Kocis, G.R., McDonald, M.K., Warrick, P.H., Feedstock routing in the ExxonMobil downstream sector. Interfaces, 41 (2), 149-163, (2011).

19. Grønhaug, R., Christiansen, M., Supply chain optimization for a liquefied natural gas business, In: Bertazzi L., van Nunen J., Speranza M.G. (Eds.), Innovations in Distribution Logistics. Lecture notes in economics and mathematical systems. Springer, pp. 195-218, (2009).

20. Grønhaug, R., Christiansen, M., Desaulniers, G., Desrosiers, J., A branch-and-price method for a liquefied natural gas inventory routing problem. Transportation Science, 44 (3), 400-415, (2010).

21. Gunnarsson, H., Rönnqvist M., Carlsson D., A combined terminal location and ship routing problem, Journal of the Operational Research Society, 57, 923-938, (2006).

22. Hwang, S.-J., Inventory constrained maritime routing and scheduling for multi-commodity liquid bulk. Ph.D. thesis, Georgia Institute of Technology, Atlanta, (2005).

23. Persson, J. A., Göthe-Lundgren, M., Shipment planning at oil refineries using column generation and valid inequalities. European Journal of Operational Research, 163 (3), 631-652, (2005).

24. Ronen, D., Marine inventory routing: Shipments planning. Journal of the Operational Research Society, 53 (1), 108-114, (2002).

25. Rakke, J., Stålhane, M., Moe, C., Andersson, H., Christiansen, M., Fagerholt K., Norstad I., A rolling horizon heuristic for creating a liquefied natural gas annual delivery program. Transportation Research C, 19 (5), 896-911, (2011).

26. Sherali, H.D., Al-Yakoob S.M, Determining an optimal fleet mix and schedules: Part II - Multiple sources and destinations, and the option of leasing transshipment depots. In Integer Programming Theory and Practice, JK Karlof (ed.), CRC Press, Boca-Raton, 167-194, (2006).

27. Sherali, H., Al-Yakoob, S., Hassan, M., Fleet management models and algorithms for an oiltanker routing and scheduling problem. IIE Transactions 31 (5), 395-406, (1999).

28. Siswanto, N., Essam, D., Sarker, R., Solving the ship inventory routing and scheduling problem with undedicated compartments. Computers \& Industrial Engineering, 61 (2), 289-299, (2011).

29. Song, J-H., Furman, K. C., A maritime inventory routing problem: Practical approach. Computers \& Operations Research, 40 (3), 657-665, (2013).

30. Stålhane, M., Rakke, J., Moe, C., Andersson, H., Christiansen M., Fagerholt. K., A Constructive and Improvement Heuristic for a Liquefied Natural Gas Inventory Routing Problem. Computers \& Industrial Engineering, 62 (1), 245-255, (2012). 